\title{
6 Bangladesch und DDR bangen um Anerkennung
}

Für das Kräfteverhältnis auf dem indischen Subkontinent und für die Stellung Indiens im Machtgefüge des Kalten Krieges bedeutete der Ausgang des kurzen Dritten Indisch-Pakistanischen Krieges gewaltige Umwälzungen. Während die indische Regierung nach dem Krieg auftrumpfen konnte, machte das verbliebene Rest-Pakistan ab Dezember 1971 eine schmerzhafte Katharsis durch und in Bangladesch begann ein Prozess der nationalen Einigung und Schaffung einer neuen, gemeinsamen Identität. Von diesem Prozess hofften auch die DDR-Außenpolitiker zu profitieren und in Bangladesch endlich ihren ersten südasiatischen Anerkennungserfolg erzielen zu können. Die Bundesregierung wiederum fürchtete die Signalwirkung eines solchen Schrittes gegenüber Indien und setzte deshalb alles daran, die Anerkennung der DDR durch Bangladesch noch zu verhindern.

\subsection{Südasiatische und globale Kriegsfolgen}

Indira Gandhis Machtstellung in Indien wurde durch den triumphalen Sieg über den Erzrivalen Pakistan dauerhaft gesichert. „Die Premierministerin ist gegenwärtig auf dem Zenith ihrer Popularität“" stellte der bundesrepublikanische Botschafter in Indien, Günter Diehl, bereits vor Kriegsende fest. Angesichts der raschen Erfolge der indischen Armee befinde sich das Land in einem „Taumel nationaler Begeisterung. “" Die indische Premierministerin umgab nun eine Aura der Unbesiegbarkeit. Selbst ein Oppositionspolitiker war nach der Kapitulation der pakistanischen Armee am 16. Dezember 1971 von Indira Gandhi hingerissen: „The name of the prime minister will go down in history as the golden sword of liberation of Bangla Desh." ${ }^{2}$

Die indische Regierung unter Indira Gandhi hatte den durch die pakistanische Innenpolitik und den Wandel im Miteinander der Großmächte entstandenen Kairos aus indischer Sicht optimal genutzt. Der verbliebene Teil Pakistans stellte für Indiens Vormachtsanspruch in Südasien keine ernstzunehmende Bedrohung mehr dar und die indische Regierung gewann deutlich an außenpolitischem Spielraum. „Das Trauma der Niederlage gegen China 1962 war überwunden“, fasste ein zeitgenössischer Beobachter die Stimmung in Indien nach Ende des

1 Diehl an AA, 13. Dezember 1971, PA AA, B 58, Bd. 608.

2 zit. n. Guha: India after Gandhi, S. 460.

D Open Access. () 2020 Alexander Benatar, publiziert von De Gruyter. @(c) BYNC-ND Dieses Werk ist lizenziert unter der Creative Commons Attribution-NonCommercial-NoDerivatives 4.0 Lizenz. 
Dritten Indisch-Pakistanischen Krieges zusammen. ${ }^{3}$ Der Sieg über Pakistan trug also erheblich zur Stärkung des indischen nationalen Selbstbewusstseins bei. ${ }^{4}$

Sowohl in indischen als auch in pakistanischen Medien wurde der Kriegsausgang bald als Sieg des Hinduismus über den Islam religiös überhöht und Indira Gandhi mitunter als hinduistische Kriegsgöttin „Durga“ stilisiert. Man hielt ihr die Teilung Pakistans ebenso zugute wie die Tatsache, sich während des Krieges gegenüber den USA behauptet zu haben. Die indische Presse hob die herausragende Bedeutung des Krieges und des Jahres 1971 überhaupt als Wendepunkt in der Geschichte des unabhängigen Indiens und insbesondere für Indira Gandhis Kongresspartei hervor. Im März 1972 konnte diese sich bei Wahlen in dreizehn teilweise kritischen indischen Bundesstaaten komfortable Mehrheiten sichern im Falle des chronisch instabilen Westbengalens allerdings weiterhin nur mithilfe massiver Einschüchterung und Wahlfälschung. ${ }^{5}$

Entgegen internationaler Befürchtungen hegte die indische Regierung außerdem keinerlei weitergehende territoriale Ansprüche im ehemaligen Ostpakistan, sondern zog ihre Truppen im Gegenteil innerhalb von drei Monaten wieder aus der Region ab, um Mujibur Rahmans gewählter Regierung das Feld zu überlassen. ${ }^{6}$

\section{Pakistanische Aufarbeitung}

Der Hausierer hatte seinen Esel mit Glasschmuck beladen, der in beiden Satteltaschen verstaut war. Ein Mann, der ihm begegnete, klopfte mit seinem Spazierstock gegen die Tasche und fragte: „Was hast Du darin?“ - „Wenn Du auch noch auf die andere Seite schlägst, habe ich nichts mehr.“

Khan Abdul Wali Khan, Präsident der National Awami Party, über die Lage Pakistans nach Kriegsende ${ }^{7}$

Für Pakistan stellte sich die Lage erwartungsgemäß völlig anders dar. Der bevölkerungsreichere Ostteil des Landes war nach einer demütigend raschen Nie-

3 Heß: Bangladesch. Tragödie einer Staatsgründung, S. 154.

4 Ebd., S. 156; Sisson / Rose: War and Secession, S. 280; Linde: Bangla Desh. Indien und die Großmächte im Pakistanischen Konflikt, S. 124.

5 Raghavan: 1971. A Global History of the Creation of Bangladesh, S. 264; McMahon: On the Periphery of a Global Conflict: India and the Cold War, 1947-1991, S. 296; Guha: India after Gandhi, S. 461-3.

6 Heß: Bangladesch. Tragödie einer Staatsgründung, S. 194; Van Schendel: A History of Bangladesh, S. 175.

7 Breuer an AA, 28. Februar 1972, PA AA, B 36, Bd. 533. 
derlage gegen den Erzfeind Indien verloren und die pakistanische Armee hatte in dem kurzen Krieg fast 6.000 Gefallene $\mathrm{zu}$ beklagen. ${ }^{8}$ Außerdem waren mehr als 90.000 pakistanische Soldaten und Polizisten in indische Gefangenschaft geraten. ${ }^{9}$ Während Indira Gandhi in Indien quer durch die politischen Lager als Siegerin gefeiert wurde, standen für die pakistanische Führung vor allem die beträchtlichen materiellen Verluste des Landes im Vordergrund.

Zwar versuchte General Yahya Khan noch am 16. Dezember 1971, dem Tag der Kapitulation, die Einheit Pakistans zu retten, indem er erklärte, wenige Tage später eine neue Verfassung mit größtmöglicher Autonomie für Ostpakistan verabschieden und dann endlich auch die lang versprochene Nationalversammlung einberufen sowie den Ostbengalen Nurul Amin zum neuen Premierminister Pakistans ernennen zu wollen. ${ }^{10}$ Seine Vorschläge fanden jedoch kein Gehör, sodass Yahya Khan am 20. Dezember 1971 dem öffentlichen politischen Druck nachgeben und vom Amt des Präsidenten zurücktreten musste. Zu seinem Nachfolger ernannte er den soeben aus den USA zurückgekehrten Zulfikar Ali Bhutto. ${ }^{11}$ Yahya Khan selbst wurde unter Hausarrest gestellt und seine Herrschaft später vom Obersten Gerichtshof in Pakistan für von Anfang an illegitim erklärt. ${ }^{12}$

Am 7. Januar 1972 entließ der neue pakistanische Regierungschef Bhutto Sheikh Mujibur Rahman aus der westpakistanischen Haft in die Freiheit, der sofort über London und Neu-Delhi in seine ostbengalische Heimat zurückkehrte. ${ }^{13}$ Bald nach Amtsantritt betonte Bhutto außerdem sein Interesse an guten Beziehungen zur UdSSR und knüpfte bereits Mitte März 1972 erste Kontakte mit der Moskauer Führung, die ebenfalls daran interessiert war, sich nicht allzu einseitig auf Indien als einzigen Ansprechpartner auf dem Subkontinent festzule-

8 Im Vergleich zu 1.500 Gefallenen auf indischer Seite, s. Matinuddin: Tragedy of Errors, S. 430-1. 9 Guha: India after Gandhi, S. 460; 462; Heß: Bangladesch. Tragödie einer Staatsgründung, S. 121; davon 65.000 Zivilisten, s. Matinuddin: Tragedy of Errors, S. 431.

10 Zulfikar Ali Bhutto würde dann dessen Stellvertreter und Außenminister. S. Linde: Bangla Desh. Indien und die Großmächte im Pakistanischen Konflikt, S. 84; wie es zwischen Bhutto und ihm schon Ende November 1971 ausgemacht worden war, s. Raghavan: 1971. A Global History of the Creation of Bangladesh, S. 233.

11 Kux: The United States and Pakistan, S. 205; Heß: Bangladesch. Tragödie einer Staatsgründung, S. 124.

12 Heß: Bangladesch. Tragödie einer Staatsgründung, S. 96.

13 Raghavan: 1971. A Global History of the Creation of Bangladesh, S. 264; Voigt: Die Indienpolitik der DDR, S. 658. Die Entscheidung, zunächst nach London zu reisen, war von Mujibur Rahman selbst ausgegangen, s. Edward Heath an Willy Brandt, 15. Januar 1972, BArch, B 136/6234. 
gen. ${ }^{14}$ Wenig später nahm die Sowjetunion auch die während des Krieges ausgesetzten Maschinenlieferungen an Pakistan wieder auf. ${ }^{15}$

Auch darüber hinaus suchte Bhutto nach neuen internationalen Partnern und bereiste noch im Januar 1972 acht arabische Staaten im Nahen und Mittleren Osten - Ausdruck einer grundlegenden regionalen Neuorientierung Pakistans stärker hin zum arabischen Raum. ${ }^{16}$ Bei einem Besuch in Peking versicherte er sich zusätzlich der pakistanischen Partnerschaft mit der VR China. Der US-Regierung unter Präsident Nixon dankte Bhutto öffentlich für ihre Unterstützung, die aus seiner Sicht zumindest einen indischen Angriff auch auf den pakistanischen Westflügel verhindert hatte. ${ }^{17}$

Zur Festigung seiner innenpolitischen Position war Bhutto an einem dauerhaften Frieden mit Indien gelegen. Auf Einladung Indira Gandhis drängte er bald zu einem bilateralen Gipfeltreffen. Im März 1972 trafen sich der pakistanische Journalist Mazhar Ali Khan und sein befreundeter indischer Kollege Sajjad Zaheer in London, um für ihre jeweiligen Regierungen die Bedingungen eines solchen Treffens und eines bilateralen Friedensvertrags auszuhandeln. Insbesondere der Status von Kaschmir blieb dabei aber weiterhin umstritten. Der Inder Zaheer forderte „an assurance that there will be no more attack, infiltration, subversion, anti-India propaganda in Kashmir" durch Pakistan. Sein pakistanischer Gesprächspartner machte jedoch deutlich, dass dies nicht Teil der offiziellen Vereinbarung werden könne: „no Government in Pak[istan] can survive if it renounces, outright, its support to Kashmiris' right of self-determination." ${ }^{18}$

Das indisch-pakistanische Treffen kam dann zwar tatsächlich im indischen Shimla zustande, doch auch Indira Gandhi und Zulfikar Ali Bhutto konnten den fundamentalen Streit um Kaschmir nicht lösen. Am 3. Juli 1972 schlossen die beiden Regierungschefs einen Friedensvertrag, in dem sie zukünftig Gewaltverzicht zugunsten von friedlichen bilateralen Verhandlungen, d.h. unter Ausschluss drittstaatlicher Intervention, vereinbarten und sich sogar auf eine Rückführung der 90.000 pakistanischen Kriegsgefangenen einigten. Über die Anerkennung der faktisch geltenden Line of Control hinaus blieb die Kaschmirfrage allerdings weiter unbeantwortet. Bhuttos mündliches Versprechen an Indira Gandhi, die

14 Heß: Bangladesch. Tragödie einer Staatsgründung, S. 160; Linde: Bangla Desh. Indien und die Großmächte im Pakistanischen Konflikt, S. 123.

15 Ebd., S. 94.

16 Sisson / Rose: War and Secession, S. 280; Heß: Bangladesch. Tragödie einer Staatsgründung, S. 159 .

17 Kux: The United States and Pakistan, S. 207-8.

18 Zit. n. Guha: India after Gandhi, S. 464. 
Line of Control alsbald auch als internationale Grenze anzuerkennen, war nur anderthalb Wochen nach der Vereinbarung von Shimla bereits wieder hinfällig. ${ }^{19}$

$\mathrm{Zu}$ sehr war die Forderung nach Kaschmir Teil der pakistanischen Identität und $\mathrm{zu}$ sehr fühlte Pakistans politische Führung sich weiterhin von ihren indischen Widersachern gedemütigt, als dass Bhutto der indischen Premierministerin gegenüber zu echten Konzessionen bereit gewesen wäre. Am 14. Juli 1972 sprach Bhutto vor der pakistanischen Nationalversammlung von einem „,solemn commitment of the people of Pakistan, that if tomorrow the people of Kashmir start a freedom movement, [...] we will be with them. “20

Die Zwei-Nationen-Theorie als Gründungs-DNA des vereinigten und unabhängigen Pakistan war durch die staatliche Teilung als unmittelbare Kriegsfolge aber widerlegt worden. ${ }^{21}$ Entsprechend wurden viele, vor allem konservative Stimmen in Pakistan laut, die sich nur schwer mit dem Verlust eines Großteils der Bevölkerung des Landes, seines Territoriums und der Erschütterung seiner eigenen Gründungsideologie abfinden konnten. Pakistanische Militärs etwa erhoben später den Vorwurf gegen Bhutto, er habe Mujibur Rahman zu früh und bedingungslos aus der Haft entlassen und den Prozess der Staatsbildung von Bangladesch dadurch unnötig beschleunigt. ${ }^{22}$ Bhutto selbst wiederum rief alle Drittländer dazu auf, die Aufnahme diplomatischer Beziehungen mit Bangladesch zu unterlassen. Er drohte, „Pakistan würde jede in diese Richtung zielende Aktion als ,feindlichen Akt' betrachten und in jedem Fall die ihm möglichen Gegenmaßnahmen treffen.“"23 Es war dies die (kurzlebige) Form einer pakistanischen Hallstein-Doktrin. ${ }^{24}$

Andererseits hatten einige andere pakistanische Politiker und Industrielle wiederum bereits vor dem Krieg hinter vorgehaltener Hand davon gesprochen, dass Ostpakistan vor allem wirtschaftlich doch mehr Last als Gewinn für das Land sei und der absehbaren Teilung entsprechend gelassen entgegengesehen.

19 Heß: Bangladesch. Tragödie einer Staatsgründung, S. 106; Guha: India after Gandhi, S. 465; Wagner: Die „verhinderte“ Großmacht? S. 107-8; Kux: The United States and Pakistan, S. 208.

20 Zit. n. Guha: India after Gandhi, S. 465.

21 Ebd., S. 466; Wagner: Die „verhinderte“ Großmacht? S. 108; Raghavan: 1971. A Global History of the Creation of Bangladesh, S. 265.

22 So etwa Niazi: The Betrayal of East Pakistan, S. 236-7.

23 Berger an AA, 28. Dezember 1971, PA AA, ISLA, Bd. 8140.

24 Gary J. Bass: Bargaining away Justice. India, Pakistan, and the International Politics of Impunity for the Bangladesh Genocide. In: International Security 41,2 (2016), S. 140-187, hier S. 158; s. auch Linde: Bangla Desh. Indien und die Großmächte im Pakistanischen Konflikt, S. 93; 104; Heß: Bangladesch. Tragödie einer Staatsgründung, S. 139; Raghavan: 1971. A Global History of the Creation of Bangladesh, S. 270. 
Vor allem viele junge Pakistanis identifizierten sich ein Vierteljahrhundert nach der Staatsgründung außerdem kaum mehr wirklich mit der Zwei-NationenTheorie. Auch manche ausländische Beobachter hegten ganz ähnliche Hoffnungen, dass Pakistans Chancen auf eine baldige demokratische Stabilisierung ohne den armen und überbevölkerten Ostflügel des Landes nun besser stehen könnten als zuvor. ${ }^{25}$

Die Aussöhnung mit dem ehemaligen Ostpakistan, dem nunmehr de facto unabhängigen Staat Bangladesch (und damit dessen offizielle Anerkennung) gestaltete sich für die pakistanische Regierung unter Zulfikar Ali Bhutto dennoch schwierig, zumal die Regierung von Bangladesch unter Mujibur Rahman bald ankündigte, pakistanische Militärs nach dem Vorbild der Nürnberger Prozesse als Kriegsverbrecher anklagen zu wollen. ${ }^{26}$

\section{Bangladesch als neuer südasiatischer Staat}

Bangladesh kann nicht mehr von der Landkarte gelöscht werden - es fragt sich, wer sich dort einzeichnet: die Demokraten, die Faschisten, die Anarchisten oder die Kommunisten. Gisela Bonn, westdeutsche Journalistin ${ }^{27}$

Eine der ersten Herausforderungen nach seiner Rückkehr aus der westpakistanischen Gefangenschaft bestand für Mujibur Rahman darin, die zersplitterten Rebellengruppen in Ostbengalen dazu zu bewegen, ihre Waffen niederzulegen. ${ }^{28}$ Bald nach Beendigung der Kampfhandlungen hatten einige von ihnen eigenmächtig mit der gewaltsamen Verfolgung vermeintlicher Kollaborateure der westpakistanischen Armee begonnen. Mujibur Rahman bemühte sich zwar, die öffentliche Ordnung wiederherzustellen und derartige Akte der Selbstjustiz zu verhindern. Dennoch wurden die Biharis in den ersten Wochen und Monaten des unabhängigen Bangladesch brutal verfolgt: Bis Mitte Mai 1972 fielen 10.000 bis 15.000 Menschen den Racheakten der bengalischen Mehrheitsbevölkerung zum

25 Ebd., S. 28; Linde: Bangla Desh. Indien und die Großmächte im Pakistanischen Konflikt, S. 91; Heß: Bangladesch. Tragödie einer Staatsgründung, S. 52-3.

26 Ebd., S. 141; Hossein: Bangladesh. Quest for Freedom and Justice, S. 127.

27 Bericht über die Reise nach Indien, Bhutan und Bangladesh vom 20. März bis zum 5. Juni 1972 von Gisela Bonn, undatiert, BArch, B 136/6234.

28 Heß: Bangladesch. Tragödie einer Staatsgründung, S. 138; Van Schendel: A History of Bangladesh, S. 177. Auch die Mukti Bahini waren mehr loser Zusammenschluss diverser paramilitärischer Gruppen als ein homogener Verband, s. Hossein: Bangladesh. Quest for Freedom and Justice, S. 126-7. 
Opfer und auch die indische Armee konnte organisierte Lynchaktionen bis zu ihrem Abzug kaum verhindern. ${ }^{29}$

Die etwa 500.000 auch nach dem Krieg in Ostbengalen verbliebenen Biharis galten in Bangladesch nun als Feinde im eigenen Land und fanden auch im früheren Westflügel Pakistans keine Aufnahme. Bhutto erklärte sich lediglich zu einem „Austausch“ bereit, indem er ebenso viele Biharis in Pakistan aufnehmen würde wie Bengalis es gen Bangladesch verließen. In Bangladesch als Verräter betrachtet, in Indien unerwünscht und in Pakistan bestenfalls geduldet, meist jedoch verachtet, waren die Biharis eine der größten Opfergruppen des Bangladeschkriegs. ${ }^{30}$

Der blutige Unabhängigkeitskampf brachte für den jüngsten Staat Südasiens weitere gewaltige gesellschaftliche Umwälzungen. Gezielte Aktionen der pakistanischen Armee im Vorfeld des Krieges hatten sich insbesondere auf die (akademische) Elite Ostbengalens sowie auf die Hindu-Minderheit konzentriert. In diesen Massakern verlor das Land einen großen Teil seiner gebildeten Schicht. Außerdem musste eine große Anzahl vergewaltigter, verwitweter und verwaister Frauen und Mädchen entstigmatisiert und gesellschaftlich reintegriert werden. ${ }^{31}$

Die politische Aufbauarbeit in Bangladesch gestaltete sich vor diesem Hintergrund alles andere als einfach. Nach Inkrafttreten einer provisorischen Verfassung am 12. Januar 1972 stand die junge Regierung von Bangladesch unter Führung Mujibur Rahmans nicht nur vor der Herausforderung, eine von Krieg und Flutkatastrophen schwer gezeichnete Nation $\mathrm{zu}$ einen, sondern sah sich zusätzlich auch mit sehr unterschiedlichen Vorstellungen darüber konfrontiert, wie diese Nation später einmal aussehen sollte. Vor allem sowjetisch unterstützte kommunistische Gruppen forderten nach der staatlichen Unabhängigkeit nun auch eine soziale Revolution für Bangladesch. ${ }^{32}$ Der erste Außenminister Bangladeschs, Abdus Samad Azad, machte jedoch bereits am 24. Januar 1972 auf einer

29 Bass: The Blood Telegram, S. 277; Heß: Bangladesch. Tragödie einer Staatsgründung, S. 143; 146.

30 Wirth / Janik / Bigler: Die Spaltung Pakistans und die Gründung der Volksrepublik Bangladesh, S. 222-3; Linde: Bangla Desh. Indien und die Großmächte im Pakistanischen Konflikt, S. 99-100; Ali: Understanding Bangladesh, S. 11; Bass: The Blood Telegram, S. 277-8; Heß: Bangladesch. Tragödie einer Staatsgründung, S. 149; Pai: The 1971 East Pakistan Genocide. A Realist Perspective, S. 10.

31 Schendel: A History of Bangladesh, S. 173; Bose: The Question of Genocide and the Quest for Justice in the 1971 War, S. 404-5.

32 Wirth / Janik / Bigler: Die Spaltung Pakistans und die Gründung der Volksrepublik Bangladesh, S. 220-1; Heß: Bangladesch. Tragödie einer Staatsgründung, S. 137-8; Van Schendel: A History of Bangladesh, S. 175. 
Pressekonferenz deutlich, dass sein Land sich weder der Sowjetunion noch dem Westen anschließen, sondern vielmehr wie Indien bündnisfrei bleiben würde. ${ }^{33}$

Doch selbst die Orientierung an Indien konnte nicht als erste politische Konstante Bangladeschs gelten. Nach Kriegsende erfolgte vielmehr eine überraschend schnelle relative Entfremdung der politischen Führung Bangladeschs von ihren indischen Befreiern. Oppositionelle Kritik an Mujibur Rahman richtete sich wiederholt gegen dessen vermeintliche Patronin Indira Gandhi und vor allem im Militär wurde der Vorwurf laut, man hätte sich im Unabhängigkeitskampf gegen die pakistanische Zentralregierung allzu sehr auf die indische Unterstützung verlassen und stünde dadurch nun unnötigerweise in der Schuld der indischen Regierung. ${ }^{34}$

Dennoch schloss Mujibur Rahman am 18. März 1972 mit der Regierung Indira Gandhi einen 25 Jahre geltenden „Freundschafts-, Zusammenarbeits- und Friedensvertrag“, der sich nicht nur nominell stark am indisch-sowjetischen Vertrag des Vorjahres orientierte. Der indischen Regierung wurden in diesem Vertrag Mitspracherechte in der Außen- und Sicherheitspolitik Bangladeschs eingeräumt. Außerdem nahm sich die Regierung von Bangladesch sowohl in ihrer Demokratie und Säkularismus bekräftigenden Verfassung als auch in der raschen Aufnahme partnerschaftlicher Beziehungen zur Sowjetunion den indischen Nachbarn zum Vorbild. ${ }^{35}$

\section{Das Echo der Großmächte}

Es war genau ein solches „Abdriften“ Bangladeschs in die sowjetische Einflusssphäre, das man in Washington fürchtete. Am 19. Januar 1972 teilte Mujibur Rahman dem westdeutschen Generalkonsul Enders in Dhaka mit, die außenpolitische Linie seiner Regierung stehe unter dem Vorbehalt vorheriger Konsultationen mit Indien und der Sowjetunion - wegen „der Bangladesh gewährten Unterstützung. “36 Diese und ähnliche Äußerungen des neuen Premierministers des nunmehr unabhängigen Bangladesch schienen die Einschätzung des USAußenministers William P. Rogers vom 28. Dezember $1971 \mathrm{zu}$ bestätigen, dass es

33 Enders an AA und Botschaft Rawalpindi, 25. Januar 1972, PA AA, ISLA, Bd. 8133.

34 Van Schendel: A History of Bangladesh, S. 182; Heß: Bangladesch. Tragödie einer Staatsgründung, S. 139-40.

35 Ebd.; Wagner: Die „verhinderte“ Großmacht? S. 118.

36 AA / IfZ: $A A P d B, 1972$, Bd. 1, Dok. 10. 
im sowjetischen Interesse sein müsste, ein unabhängiges Bangladesch zu schaffen, das unter seinem und Indiens Einfluss stehen würde. ${ }^{37}$

In Indien wurde die unaufdringliche Hilfe der UdSSR im Dritten IndischPakistanischen Krieg ebenfalls mit Wohlwollen aufgenommen. Insofern bedeutete der indische Sieg gegen den US-Verbündeten Pakistan - wie von Nixon und Kissinger befürchtet - tatsächlich auch einen sowjetischen Erfolg im globalen Ringen der Großmächte um Einfluss in der Dritten Welt. ${ }^{38}$ Die schmachvolle Niederlage seines südasiatischen Partners empfand der US-Präsident durchaus auch als persönliches Scheitern. Von Indira Gandhi sprach er nach Kriegsende als „the bitch“, „old witch“ und schimpfte: „The Indians are bastards anyway.“39

Kissinger und er hielten sich allerdings zugute, durch ihren Druck auf die indische Regierung immerhin verhindert $\mathrm{zu}$ haben, dass Indira Gandhis Armee auch noch den pakistanischen Westflügel zerschlug. ${ }^{40}$ In öffentlichen Stellungnahmen bemühte sich US-Außenminister Rogers, Indien als Aggressor im Krieg um Ostbengalen darzustellen: „the Government of India encouraged and supported an insurgent movement whose principal purpose was the creation of an independent state of Bangladesh by force of arms. “ Dadurch sei eine der größten humanitären Katastrophen der Gegenwart entstanden. Im Übrigen seien die Verhältnisse in und um Ostpakistan überhaupt unklar gewesen und Gräueltaten seien von beiden Seiten des Konflikts verübt worden, versuchte er die überaus kontroverse Haltung seiner Regierung im Nachhinein zu rechtfertigen. ${ }^{41}$ Auch der amerikanische Chefdiplomat war nach Kriegsende also auf die Linie Nixons und Kissingers eingeschwenkt und stellte sich auf die Seite der pakistanischen Zentralregierung.

Nur wenige Monate nach Beginn des Wandels in den amerikanischchinesischen Beziehungen hatten die VR China und die USA im Bangladeschkrieg zum ersten Mal vereint gegen den gemeinsamen Gegner Sowjetunion gestanden. Nixon und Zhou Enlai äußerten sich später gleichermaßen dankbar für die zuverlässige Maklerrolle, die Yahya Khan in der Annäherung der beiden vormals verfeindeten Staaten gespielt hatte. Die amerikanisch-chinesische Eintracht währte jedoch nicht allzu lang: Beim historischen ersten Treffen eines US-Präsiden-

37 AA / IfZ: $A A P d B, 1971$, Bd. 3, Dok. 451.

38 Wagner: Die „verhinderte“ Großmacht? S. 209; Heß: Bangladesch. Tragödie einer Staatsgründung, S. 9.

39 Zit. n. Guha: India after Gandhi, S. 460.

40 Kux: The United States and Pakistan, S. 205; Heß: Bangladesch. Tragödie einer Staatsgründung, S. 134-5.

41 Rogers: United States Foreign Policy 1971, S. 110; Bose: Dead Reckoning. Memories of the 1971 Bangladesh War, S. 72. 
ten mit Mao Zedong im Februar 1972 zeigte dieser Nixon gegenüber kaum die gewünschte Dankbarkeit. ${ }^{42}$

Die Annäherung an die VR China brachte der US-Regierung also nicht den von Nixon und Kissinger erhofften Schlüssel zu einem gesichtswahrenden Ende des blutigen Vietnamkriegs. Bald machte die chinesische Seite im Gegenteil deutlich, dass sie ihrem ideologisch verbündeten Brudervolk in Nordvietnam nicht ohne Weiteres in den Rücken fallen würde. ${ }^{43}$ Im April 1972 sah die US-Regierung den von Indien geschaffenen Tatsachen dennoch ins Auge und erkannte Bangladesch als souveränen Staat an. Die sowjetische Führung hatte diesen Schritt bereits am 25. Januar 1972 getan. $^{44}$

\subsection{Die DDR auf dem Weg zur Anerkennung}

Während sich die Folgen des kurzen Dritten Indisch-Pakistanischen Krieges in Südasien bald manifestierten und die weltweite Akzeptanz der maßgeblich durch die indische Armee in Ostbengalen geschaffenen Tatsachen nur noch eine Frage der Zeit zu sein schien, begann für die beiden deutschen Staaten nach Ende des Krieges das eigentliche Ringen um ihre jeweiligen deutschlandpolitischen Interessen in den nunmehr drei Nachfolgestaaten Britisch-Indiens. Zwar lag das Hauptaugenmerk beiderseits der Mauer weiterhin auf der Haltung Indiens gegenüber dem Ost-Berliner Drängen auf Anerkennung, doch auch alle Schritte der Regierungen Pakistans und Bangladeschs wurden von den politischen Führungen in BRD und DDR in den Wochen und Monaten nach Kriegsende sehr genau verfolgt.

42 Heß: Bangladesch. Tragödie einer Staatsgründung, S. 130; Macmillan: Nixon and Mao, S. 72; 238.

43 Ebd., S. 266-7; Xia / Tudda: Beijing, 1972, S. 59. Zumindest indirekt half die Annäherung den USA dennoch: Mao riet den Nordvietnamesen, sich mit dem südvietnamesischen Präsidenten Thieu zu arrangieren. Sobald die USA ihre Truppen abgezogen hätten und Ruhe eingekehrt sein würde, könne man den Kampf mit dem Süden wieder aufnehmen und gewinnen. Genau so kam es 1975 dann auch. S. Macmillan: Nixon and Mao, S. 271.

44 Moses: Die Vereinten Nationen, humanitäres Engagement und die Menschenrechte, S. 361; Prasad: Indo-Soviet Relations 1947-1972, S. 386. 


\section{Hoffnungen der DDR in Südasien}

Obwohl die sowjetische Führung auch unmittelbar nach Ende der Kriegshandlungen in Ostbengalen noch immer Einwände gegen eine sofortige Anerkennung Bangladeschs durch die DDR erhob, ${ }^{45}$ versuchten DDR-Vertreter ihre Unterstützung für Indien in diesem Konflikt nun besonders herauszustellen, in der Hoffnung, die indische Regierung dadurch endlich zur diplomatischen Anerkennung zu bewegen. ${ }^{46}$

In einem Brief an Indira Gandhi gab Willi Stoph am 18. Dezember 1971, zwei Tage nach der pakistanischen Kapitulation, der pakistanischen Zentralregierung die Hauptschuld am Ausbruch des Dritten Indisch-Pakistanischen Krieges. Er versicherte der indischen Regierungschefin, die Regierung der DDR habe größtes Verständnis dafür, dass Indien aufgrund der „kolonialen Unterdrückung und des Völkermordes des pakistanischen Militärregimes gegenüber dem Volk von Bangla Desh“ zu einem militärischen Eingreifen in den Konflikt praktisch gezwungen gewesen war. Bereits in diesem Schreiben stellte Stoph außerdem fest, dass „die Regierung der DDR die rechtmäßigen Repräsentanten des Volkes von Bangla Desh“ anerkenne und verlieh seiner Gewissheit Ausdruck, „daß sich die freundschaftlichen staatlichen Beziehungen zwischen der DDR und der Republik Indien [...] in nächster Zukunft erfolgreich weiterentwickeln werden. “47 Allem Anschein nach hoffte der DDR-Ministerratsvorsitzende, eine mögliche Anerkennung Bangladeschs durch die DDR würde zu einer Anerkennung wiederum der DDR durch Indien führen.

Im indischen Außenministerium durchschaute man dieses Ost-Berliner Kalkül zwar sehr wohl, der indische Generalkonsul in Ost-Berlin, J. C. Ajmani, gestand seinem Gastland jedoch später immerhin $\mathrm{zu}$, in der Unterstützung Indiens während des Bangladeschkriegs „nicht ausschließlich opportunistisch“ gehandelt $\mathrm{zu}$ haben. ${ }^{48}$ Bald nach Kriegsende bekräftigte ein indischer Diplomat auch gegenüber dem DDR-Generalkonsul Herbert Fischer, die „indische Seite [sei] der Meinung, daß [in der Frage der DDR-Anerkennung] längeres Warten

45 Antwort aus Moskau, „durchgesagt vom Genossen Gorinowitsch“, dem damaligen sowjetischen Botschafter in der DDR, 17. Dezember 1971, PA AA, M 1, C 1046/77.

46 Voigt: Die Indienpolitik der DDR, S. 644; Schneider: Die Außenpolitik der DDR gegenüber Südasien, S. 20; so auch später DDR-Außenminister Otto Winzer gegenüber dem sowjetischen Außenminister Andrej Gromyko, s. „Niederschrift über die Unterredung zwischen Genossen Winzer und Genossen Gromyko am 19. Januar 1972“, 20. Januar 1972, BArch, DY 30/11654.

47 Willi Stoph an Indira Gandhi, 18. Dezember 1971, PA AA, M 1, C 1762/76.

48 „Annual Political Report for 1971“ von J. C. Ajmani, undatiert, NAI, Ministry of External Affairs, $\mathrm{H} \mathrm{I} / 1011(55) / 72$. 
unzweckmäßig“ sei. ${ }^{49}$ Aus ostdeutscher Perspektive schien der Zeitpunkt also günstig, der indischen Regierung einen Gefallen zu tun. Herbert Fischer bemühte sich, dieses Momentum zu nutzen, indem er Indira Gandhi noch einmal persönlich zu überzeugen versuchte, dass der Zeitpunkt für die Herstellung diplomatischer Beziehungen zwischen Indien und der DDR nun gekommen sei. Indira Gandhi ging hierauf jedoch nicht ein. ${ }^{50}$

Aus dem MfS kamen in diesen Tagen ebenfalls eher ernüchternde Nachrichten. Durch ihr Informandennetzwerk erfuhren Mitarbeiter des DDR-Geheimdienstes, dass die indische Regierung inzwischen nicht mehr primär nach politischer Unterstützung für das befreite Ostbengalen suchte, sondern vor allem nach Wirtschaftshilfe. Und die war eher aus Bonn als aus Ost-Berlin zu erwarten. Insofern „verliere jene Kombination an Bedeutung, wonach Indien die DDR und die DDR als Gegenleistung Bangla Desh völkerrechtlich anerkennen werde“, hieß es Ende Dezember 1971 in einer Analyse des MfS. ${ }^{51}$

Die DDR-Vertretung in Neu-Delhi verfiel daraufhin in Aktionismus. Ihre bereits vielfältigen Maßnahmen gegenüber der indischen Regierung flankierte sie nun zusätzlich mit dem durchaus erfolgreichen Versuch, auch eine große Anzahl indischer Parlamentarier für ihre Sache zu gewinnen. Am 23. Dezember 1971 überreichte eine Delegation von dreizehn Abgeordneten der indischen Premierministerin eine von insgesamt 447 Mitgliedern beider Häuser des indischen Zentralparlaments sowie 468 Abgeordneten aus vier Unionsstaatenparlamenten unterzeichnete Petition, in der diese die unverzügliche Herstellung diplomatischer Beziehungen mit der DDR verlangten. Begründet wurde diese Forderung damit, dass die DDR nicht nur großzügige Hilfe für Bangladesch-Flüchtlinge in Indien geleistet, sondern sich auch als erstes Drittland im Sinne der ostbengalischen Bevölkerung für eine politische Lösung des Konflikts um Ostbengalen eingesetzt habe. Die Bundesregierung habe demgegenüber eine ausgesprochen Indien-feindliche Haltung eingenommen. ${ }^{52}$

Hiervon zeigte die indische Regierung sich dann doch beeindruckt, wie nun auch die bundesrepublikanische Seite erfahren musste. Als am 29. Dezember 1971 ein westdeutscher Botschaftsgesandter dem indischen Joint Secretary Dr. S. Sinha ein Schreiben überreichte, in dem die Bundesregierung Indien für seine

49 Herbert Fischer an Axen, Winzer, Fischer und Radde, 28. Dezember 1971, BArch, DY 30/97822. 50 Herbert Fischer an Axen, Winzer und Radde, 23. Dezember 1971, BArch, DY 30/97822.

51 „Information über Indiens politische Position gegenüber Pakistan und Bangla Desh sowie über die Haltung einzelner Regierungen gegenüber den Staaten auf den indischen Subkontinent“, 24. Dezember 1971, BStU, MfS, HV A, Nr. 398.

52 Voigt: Die Indienpolitik der DDR, S. 664; s. Wortlaut der Petition, unterzeichnet von N. L. Gupta, undatiert, BArch, B 136/6262. 
Zurückhaltung in der Frage der DDR-Anerkennung und damit seinen Beitrag zur Détente in Europa dankte, ${ }^{53}$ erläuterte Sinha seinem Gesprächspartner, die DDR habe sich in den letzten Wochen sehr geschickt verhalten und „eine große Zahl einflußreicher Abgeordneter überzeugt, daß die DDR zur Anerkennung von Bangladesh bereit sei und daß sie im Unterschied zur Bundesrepublik eindeutig und vorbehaltlos auf Seiten Indiens stehe. “54

Vor allem die Tatsache, dass die DDR keinerlei offizielle Beziehungen $\mathrm{zu}$ Pakistan unterhielt, wusste das DDR-Generalkonsulat sehr wirksam als Beweis für die immer wieder herausgestellte pro-indische und anti-pakistanische Haltung der DDR zu nutzen, musste man in der bundesrepublikanischen Botschaft feststellen. ${ }^{55}$ Außerdem, so Sinha, seien die westdeutschen Bemühungen um den Abschluss eines Generalvertrages zur Regelung der deutsch-deutschen Beziehungen ohnehin aussichtslos und insofern sei nicht einzusehen, warum eine Anerkennung der DDR durch Indien die bundesrepublikanische Entspannungspolitik weiterhin behindern würde. ${ }^{56}$ Die schlimmsten Befürchtungen der westdeutschen Botschaft in Neu-Delhi, dass Indien die DDR nun tatsächlich bald anerkennen könnte, schienen sich zu bestätigen. ${ }^{57}$

Entgegen früherer Zusagen der indischen Regierung rechnete der bundesrepublikanische Botschafter Diehl nun mit einer DDR-Anerkennung bereits Ende Januar oder Anfang Februar 1972. Diese neu entstandene Lage führte er vor allem auf das geschickte Agieren der DDR-Außenpolitiker im Umgang mit der Bangladesch-Frage zurück. Diehl empfahl der Bundesregierung, so bald wie möglich bilaterale Regierungskonsultationen anzuberaumen, den indischen Botschafter in Bonn vorzuladen sowie nicht zuletzt mit den europäischen Partnern und Großbritannien über eine rasche Anerkennung Bangladeschs zu beraten, ${ }^{58}$ um der DDR so den Wind aus den Segeln zu nehmen.

Diehls Amtskollege in Pakistan, Norbert Berger, warnte demgegenüber ausdrücklich vor den Konsequenzen eines solchen Schrittes in seinem Gastland. Wenn die Bundesregierung Bangladesch anerkenne, müsse sie damit rechnen, „daß Pakistan ggf. unseren Wünschen im Bereich der Deutschland-Politik künftig nicht mehr Rechnung tragen wird. Eine spürbare Verschlechterung der bisher normalen bis freundlichen bilateralen Beziehungen wäre in jedem Falle unver-

53 AA / IfZ: $A A P d B$, Bd. 3, Dok. 454, Anm. 3.

54 AA / IfZ: $A A P d B, 1971$, Bd. 3, Dok. 454, Anm. 9.

55 Ebd.

56 AA / IfZ: $A A P d B, 1971$, Bd. 3, Dok. 454.

57 Vgl. am Vortag noch Diehl an AA, Botschaften Rawalpindi und Moskau sowie die SV bei der UNO in New York, 28. Dezember 1971, PA AA, B 37, Bd. 637.

58 AA / IfZ: $A A P d B$, 1971, Bd. 3, Dok. 454. 
meidlich." Er gab außerdem zu bedenken, dass durch eine solche Entscheidung auch die arabischen Bündnispartner Pakistans vor den Kopf gestoßen werden könnten. Insgesamt riet Berger also von einer schnellen Anerkennung Bangladeschs durch die Bundesregierung ab. Seines Erachtens sollte sie „erst nach Konsolidierung der dortigen Verhältnisse und möglichst in zeitlicher Abstimmung mit den übrigen europäischen Ländern, den USA und Japan erfolgen. “59

Im Generalkonsulat der DDR erahnte man die Überlegungen der Gegenseite und war besorgt um den positiven Effekt der eigenen Anerkennung Bangladeschs. Herbert Fischer hielt es am 30. Dezember 1971 in einem Bericht an seine OstBerliner Vorgesetzten „für unbedingt erforderlich, dass [die] DDR schnell handelt und vor Anderen [die] Anerkennung [Bangladeschs] ausspricht."60 Von Vertretern der sozialistischen Bruderstaaten hatten DDR-Diplomaten zudem erfahren müssen, dass der angestrebte Automatismus „Anerkennung zum Preis der Anerkennung “ keineswegs gesichert war. ${ }^{61}$ Der DDR-Außenminister Otto Winzer vermutete daraufhin, Indira Gandhis engste Ratgeber rieten ihr noch immer dazu, die Wünsche der Bundesrepublik zu berücksichtigen. ${ }^{62}$ Die ostdeutschen Diplomaten in Neu-Delhi reagierten daraufhin panisch. In einem persönlichen Brief an Indira Gandhis engen Vertrauten P. N. Haksar behauptete der DDR-Generalkonsul Herbert Fischer am 6. Januar 1972 pauschal: „[Mr. Brandt’s] demand that negotiations between the FRG and the GDR should not be disturbed by third countries recognizing the GDR seems to [have] be[en] initiated by Mr. Bhutto!“63

Abgesehen davon, dass ihr Erfolg mehr als zweifelhaft war, bestand für eine mögliche Anerkennung Bangladeschs durch die DDR zu diesem Zeitpunkt noch immer eine weitere Hürde. Das MfAA war in dieser Frage zwingend auf das Moskauer Plazet angewiesen ${ }^{64}$ und den sowjetischen Außenpolitikern war deutlich weniger an einer sofortigen Anerkennung Bangladeschs gelegen als der OstBerliner DDR-Führung. In Moskau bedauerte man die internationale Isolation, zu der das wiederholte sowjetische Veto zugunsten Indiens im UN-Sicherheitsrat während des Krieges geführt hatte. Durch eine verfrühte Anerkennung der von der indischen Armee gewaltsam geschaffenen Fakten in Ostbengalen wollte die

59 Berger an AA und Generalkonsulat Dacca, 16. Dezember 1971, PA AA, DHAK, Bd. 9092.

60 Herbert Fischer an Axen, Winzer, Fischer und Radde, 30. Dezember 1971, BArch, DY 30/97822. 61 „Vermerk über ein Gespräch mit dem Botschafter der VR Polen, Genossen Gede, am 27.12.1971“ von Moldt, 27. Dezember 1971, PA AA, M 1, C 1052/77.

62 Voigt: Die Indienpolitik der DDR, S. 660.

63 Herbert Fischer an P. N. Haksar, 6. Januar 1972, NMML, P. N. Haksar Papers (I-II Installment), Correspondence with Fischer, Herbert.

64 „Zur Frage der Herstellung diplomatischer Beziehungen DDR - Bangla Desh“, 15. Dezember 1971, BArch, DY 30/97788; vgl. Voigt: Die Indienpolitik der DDR, S. 655. 
Moskauer Führung diese Isolation nun nicht noch zusätzlich unterstreichen. Im Übrigen glaubten auch sowjetische Diplomaten kaum, dass es der DDR-Führung gelingen würde, die indische Regierung davon zu überzeugen, dass die DDR sich im Bangladeschkrieg wesentlich loyaler verhalten hatte als die BRD. ${ }^{65}$ Dennoch gaben UdSSR-Vertreter westdeutschen Diplomaten unmittelbar nach Kriegsende $\mathrm{zu}$ verstehen, dass die Ost-Berliner DDR-Führung Bangladesch möglicherweise bald anerkennen könnte. ${ }^{66}$

In der bundesrepublikanischen Botschaft in Neu-Delhi fürchtete man nach dieser sowjetischen Mitteilung sehr konkret die bevorstehende Anerkennung Bangladeschs durch die DDR und die Konsequenzen, die dies seitens der indischen Regierung möglicherweise nach sich ziehen könnte. Es sei in diesem Falle ,aus indischer Sicht innen- und außenpolitisch nicht mehr einleuchtend zu begründen, warum Indien selbst und die DDR sich nicht anerkennen sollten“, glaubte der bundesrepublikanische Botschafter in Neu-Delhi. ${ }^{67}$ Westdeutsche Diplomaten suchten in diesen Tagen also verstärkt nach Argumenten, um der intensiven DDR-Aktivität auf dem indischen Subkontinent etwas entgegenzusetzen. Das Generalkonsulat in Kalkutta bat daher um detaillierte Informationen zur westdeutschen Hilfe für inzwischen nach Ostbengalen zurückgekehrte Flüchtlinge und empfahl der Bonner AA-Zentrale, in öffentlichen Mitteilungen zu betonen, „dass wir die Bangladesh-Bevölkerung bereits seit vielen Monaten effektiv unterstützt haben." 68

Außerhalb Südasiens beurteilte man die deutschlandpolitische Lage vor Ort deutlich weniger dramatisch. So schätzte etwa der Vertreter der Bundesregierung bei der UNO in New York unmittelbar nach Kriegsende ein: „eine Anerkennung der DDR durch Indien würde hier zurzeit als Preis für die Unterstützung der Sowjetunion angesehen werden und eher gegen Indien und gegen die DDR wirken. Der Einfluss Indiens in der Dritten Welt ist durch das Vorgehen in Ostpakistan tief gesunken, so dass aus hiesiger Sicht eine Kettenreaktion [nach einer indischen DDR-Anerkennung] gegenwärtig kaum zu erwarten ist.“ Er gab außerdem zu bedenken, dass sich auch die Bundesregierung ,durch eine frühe Anerkennung von Bangla Desh in den Vereinten Nationen kaum Freunde erwerben [...], aber viele vor den Kopf stoßen“ würde. ${ }^{69}$

65 „Zur Frage der Anerkennung Bangla Deshs“, undatiert, BArch, DY 30/97788.

66 Diehl an AA sowie Botschaften Moskau und Rawalpindi, 17. Dezember 1971, PA AA, ISLA, Bd. 8130.

67 Diehl an AA, 7. Januar 1972, PA AA, B 37, Bd. 666.

68 Linsser an AA und Botschaft Neu-Delhi, 10. Januar 1972, PA AA, B 30, Bd. 671.

69 Gehlhoff an AA sowie Botschaften Washington, London, Paris, Neu-Delhi, Rawalpindi, Rom, Moskau, Tokio und die SV bei der NATO in Brüssel, 17. Dezember 1971, PA AA, ISLA, Bd. 8130. 


\section{Die DDR und Bangladesch}

In Ost-Berlin hatte man sich mittlerweile entschieden: Da Vertreter Bangladeschs ostdeutschen Diplomaten schon früher eine gegenseitige Anerkennung zugesagt hatten, würde die DDR zunächst Bangladesch anerkennen. ${ }^{70}$ So konnte man sich der Anerkennung zumindest eines südasiatischen Staates gewiss sein und darüber hinaus bestand noch immer Hoffnung, dass dieser Schritt auch auf die indische Regierung die gewünschte Wirkung haben würde. ${ }^{71}$

Als eine Art Testballon teilte Herbert Fischer dem Leiter der Mission Bangladeschs in Neu-Delhi, H. R. Choudhury, am 17. Dezember 1971 mit, dass die DDR die im Dezember 1970 gewählten Abgeordneten ,als die einzig rechtmäßigen und kompetenten Vertreter von Bangla Desh" betrachte. ${ }^{72}$ Fischers Erklärung wurde positiv entgegengenommen. Choudhury bekräftigte noch einmal das bereits zuvor gegebene Versprechen, dass im Falle einer Anerkennung Bangladeschs durch die DDR Gegenseitigkeit gelten würde und vergaß dabei nicht zu erwähnen, dass bundesrepublikanische Diplomaten bereits vor einer Anerkennung der DDR durch Bangladesch gewarnt hätten. ${ }^{73}$

Im MfAA drängte man daraufhin zu noch größerer Eile. Am 22. Dezember 1971 wurde ein Anlass gefunden, einen offiziellen DDR-Vertreter nach Ostbengalen zu entsenden. Sachspenden (Medikamente, Schulmaterialien etc.) sollten mit einer Sondermaschine der DDR-Fluggesellschaft „Interflug“ nach Dhaka gebracht und dieser Transport von einem ostdeutschen Diplomaten begleitet werden. Außerdem wollte man bei dieser Gelegenheit öffentlichkeitswirksam 25 erholungsbedürftige Ostbengalen für vier Wochen zur Behandlung in die DDR einladen. ${ }^{74}$

70 „Vermerk über Gespräch mit dem Außenminister von Bangla Desh, Abdus Samad Azad am 7. 1.1972, 10.30 Uhr bis 11.00 Uhr im Ashoka Hotel“ von Herbert Fischer, 8. Januar 1972. PA AA, M 1, C 787/75; anberaumt schon zuvor, s. Herbert Fischer an Winzer und Radde, 8. Dezember 1971, BArch, DY 30/97822; s. auch „Zur Frage der Herstellung diplomatischer Beziehungen DDR Bangla Desh“, 15. Dezember 1971, BArch, DY 30/97788.

71 „Vermerk über ein Gespräch des Stellvertreters des Ministers für Auswärtige Angelegenheiten der DDR, Genossen Oskar Fischer mit dem Gesandten der Botschaft der UdSSR in der DDR, Genossen Gorinowitsch, am 3. 1. 1972“ von Bauer, 3. Januar 1972, PA AA, M 1, C 787/75; „Vorschlag zum weiteren Vorgehen gegenüber Indien und Bangla Desh“ von Radde, 4. Januar 1972, PA AA, M 1, C 787/75.

72 Notiz vom 17. Dezember 1971, BArch, DY 30/97788.

73 „Zu den Beziehungen DDR - Indien und zum Konflikt auf dem indischen Subkontinent“, Bericht des Generalkonsulats Neu-Delhi, undatiert, BArch, DY 30/97822.

74 „Beschluß über eine Spende der Deutschen Demokratischen Republik an die Regierung von Bangla Desh“, Präsidium des Ministerrates, 22. Dezember 1971, BArch, DC 20-I/4/2577. 
Während DDR-Außenpolitiker alles daransetzten, die gegenseitige Anerkennung mit Bangladesch zu sichern, war man auf bundesrepublikanischer Seite nach eigenen Informationen aus der Bangladesch-Mission in Neu-Delhi recht zuversichtlich, dass der DDR selbst dies nicht gelingen würde. „Den Vertretern von ,Bangla Desh' scheint - schon aus wirtschaftlichen Gründen - mehr an einer Anerkennung durch die Bundesrepublik gelegen zu sein“75, hieß es bald nach Kriegsende in einem AA-Bericht. Dennoch wollte man auf eine britische Anregung, die Entwicklungshilfezahlungen an das ehemalige Ostpakistan nach Kriegsende nun fortzuführen, zunächst noch nicht eingehen, um dieses später womöglich noch wichtige Druckmittel nicht zu früh aus der Hand zu geben. ${ }^{76}$

Bundesrepublikanische Diplomaten sprachen in diesen Tagen wiederholt und nicht ohne Erfolg in der Bangladesch-Mission in Neu-Delhi vor, um vor einer DDR-Anerkennung zu warnen. Am 29. Dezember 1971 gab der Missionsleiter Choudhury einem westdeutschen Diplomaten gegenüber freimütig zu, der DDRGeneralkonsul Herbert Fischer habe ihm zwar eine gegenseitige Anerkennung vorgeschlagen, er werde seiner Regierung jedoch empfehlen, dieses Angebot selbst im Falle einer Anerkennung durch die DDR nicht anzunehmen. In der Regel folge die Regierung seinen Empfehlungen auch. ${ }^{77}$ Eine Fehleinschätzung, wie sich später herausstellen sollte.

Das Auswärtige Amt bemühte sich, diese Empfehlung Choudhurys durch sanften Druck auf die kurz zuvor in Dhaka offiziell gebildete Regierung zu unterstreichen. Um den befürchteten Effekt einer Anerkennung Bangladeschs durch die DDR abzumildern, wurde der dortige westdeutsche Generalkonsul Rolf Enders Anfang Januar 1972 angewiesen, vor Ort „die notwendigen Kontakte unterhalb der Schwelle der Anerkennung aufzunehmen. " 78 Außerdem erwog man im AA, einige Entwicklungshilfeprojekte in Ostbengalen bereits früher als geplant wieder aufzunehmen. ${ }^{79}$ Botschafter Diehl in Neu-Delhi hoffte, solche Gesten könnten eine Anerkennung der DDR durch Bangladesch womöglich noch abwenden und empfahl der Bundesregierung noch einmal, Bangladesch selbst sobald wie möglich und gemeinsam mit anderen europäischen Partnerländern anzuerkennen. ${ }^{80}$

75 Müller an Botschaft London, 20. Dezember 1971, PA AA, B 37, Bd. 633.

76 Jungfleisch an Botschaften Brüssel, Ottawa, Paris, Rom, Tokio, Den Haag, Oslo, Stockholm, Washington, London, Kopenhagen, Wien, Islamabad und Neu-Delhi sowie die SV bei der UNO in New York, 23. Dezember 1971, PA AA, B 30, Bd. 671.

77 Diehl an AA, 31. Dezember 1971, PA AA, B 37, Bd. 637.

78 Vermerk von Berendonck, 4. Januar 1972, PA AA, B 37, Bd. 691.

79 Vermerk von Herbst, 6. Januar 1972, PA AA, B 30, Bd. 671.

80 Diehl an AA sowie Botschaften Rawalpindi, Moskau, Paris und London, 7. Januar 1972, PA AA, B 30, Bd. 673. 
Zwischen den Diplomaten der BRD und der DDR begann ein regelrechter „Wettlauf nach Dhaka. “81 Im MfAA fürchteten DDR-Diplomaten die Wankelmütigkeit der unerfahrenen Regierung von Bangladesch. Trotz Zusage des designierten Außenministers von Bangladesch bezweifelte der stellvertretende DDR-Außenminister Oskar Fischer am 3. Januar 1972, dass bei einer Anerkennung durch die DDR wie vereinbart Gegenseitigkeit gelten würde. Er hatte anderslautende Informationen erhalten und vermutete richtig, „dass hier bereits Westdeutschland seine Hände im Spiel“ hatte. ${ }^{82}$ Der oberste Vertreter der DDR in Indien Herbert Fischer war dagegen deutlich zuversichtlicher, dass die Regierung von Bangladesch sich an die gegebene Zusage halten würde. ${ }^{83}$

Die Begleitung der DDR-Hilfssendung nach Südasien war in Ost-Berlin mittlerweile zur Chefsache geworden. Otto Winzer, der Außenminister der DDR, würde den selbst Transport nach Südasien begleiten und hoffte, bei einer Zwischenlandung in Neu-Delhi auch mit Indira Gandhi und seinem indischen Amtskollegen Swaran Singh sprechen zu können. ${ }^{84}$ Herbert Fischer drängte seinen Vorgesetzten, die Reise nach Indien nicht unnötig hinauszuzögern, da ihre Erfolgsaussichten sich sonst aufgrund weiterer Interventionen der bundesrepublikanischen Botschaft verschlechtern könnten. ${ }^{85}$ Vor der Abreise Winzers nach Südasien bedurfte es allerdings nach wie vor einer sowjetischen Zustimmung zur Anerkennung Bangladeschs durch die DDR. ${ }^{86}$

Am 7. Januar 1972 kam endlich die Nachricht aus dem Politbüro der KPdSU in Moskau, dass man die Haltung der DDR gegenüber Bangladesch als sehr verständlich betrachte und sie nötigenfalls sogar in der indischen Öffentlichkeit unterstützen würde. ${ }^{87}$ Am selben Tag traf der DDR-Generalkonsul Herbert Fischer den designierten Außenminister Bangladeschs Abdus Samad Azad, um sicherzugehen, dass die versprochene Gegenseitigkeit der Anerkennung weiterhin gelten

81 Voigt: Die Indienpolitik der DDR, S. 660.

82 „Vermerk über ein Gespräch des Stellvertreters des Ministers für Auswärtige Angelegenheiten der DDR, Genossen Oskar Fischer mit dem Gesandten der Botschaft der UdSSR in der DDR, Genossen Gorinowitsch, am 3. 1. 1972“" von Bauer, 3. Januar 1972, PA AA, M 1, C 787/75.

83 Herbert Fischer an Otto Winzer, 4. Januar 1972, BArch, DC 20/17218.

84 Otto Winzer an Herbert Fischer, 5. Januar 1972, BArch, DC 20/17216.

85 Herbert Fischer an Otto Winzer, 5. Januar 1972, BArch, DC 20/17216.

86 „Vermerk über ein Gespräch des Stellvertreters des Ministers für Auswärtige Angelegenheiten der DDR, Genossen Oskar Fischer mit dem Gesandten der Botschaft der UdSSR in der DDR, Genossen Gorinowitsch, am 3. 1. 1972“ von Bauer, 3. Januar 1972, PA AA, M 1, C 787/75; „Vorschlag zum weiteren Vorgehen gegenüber Indien und Bangla Desh“ von Radde, 4. Januar 1972, PA AA, M 1, C 787/75.

87 „Telefonische Mitteilung des Genossen Erich Honecker“, 7. Januar 1972, PA AA, M 1, C 787/75; anders interpretiert dies allerdings Voigt: Die Indienpolitik der DDR, S. 656; 659. 
würde. Azad sagte dies grundsätzlich zu, äußerte in dem Gespräch allerdings bereits Bedenken hinsichtlich der bundesrepublikanischen Reaktion. Herbert Fischer wischte diese rasch beiseite: Die Herstellung diplomatischer Beziehungen zwischen der DDR und Bangladesch sei für die von Bangladesch ebenfalls angestrebte Anerkennung durch die Bundesrepublik Deutschland im Gegenteil eher noch förderlich. Er erläuterte Azad, „dass überall, wo die DDR allein vertreten ist, die Bundesrepublik bemüht ist, möglichst schnell ebenfalls in Erscheinung zu treten. [...] Dieses Argument fand der Minister sehr einleuchtend“ und stimmte dem geplanten Besuch des DDR-Außenministers in Dhaka am 12. Januar $1972 \mathrm{zu}^{88}$

Am Folgetag, dem 8. Januar 1972, bat Azad Herbert Fischer dann jedoch unvermittelt um einen Aufschub des Besuchs um einige Tage. Seines Erachtens war es nunmehr am besten, „wenn der Minister am 15. und 16.1. in Dacca sein könnte.“ Fischer vermutete daraufhin, „dass ihm von indischer Seite empfohlen wird, den Termin etwas hinauszuschieben. “89 DDR-Diplomaten in Dhaka nahmen später an, diese Verzögerung durch Azad sei außerdem auf den Wunsch der Regierung von Bangladesch zurückzuführen, ihre Bündnisfreiheit hervorzuheben, indem sie nach Ende des Krieges zunächst die Anerkennung durch einige westliche Länder abwartete. ${ }^{90}$ Von Azad erfuhren sie zudem, dass Mujibur Rahman unbedingt vermeiden wollte, die Bundesregierung unnötig zu verärgern, da er auf einen positiven Einfluss der Regierung Brandt auf die EWG und den Commonwealth hoffte. Ernüchtert mussten die ostdeutschen Diplomaten in Dhaka feststellen: „R[ahman] versucht bereits jetzt günstige Voraussetzungen für ein ,Balancieren’ zwischen BRD und DDR zu schaffen.“91

Trotz dieser Irritationen traf Otto Winzer am 9. Januar 1972 wie geplant in Neu-Delhi ein. Am Flughafen empfing ihn der indische Minister für Tourismus und Zivilluftfahrt Dr. Karan Singh. Das indische Außenministerium gab zwar zunächst an, selbst erst am Vorabend über diesen „unscheduled visit“ informiert worden zu sein, korrigierte diese offizielle Aussage später jedoch dahingehend, dass der DDR-Außenminister „Gast der indischen Regierung sei und eine schon vor längerer Zeit ausgesprochene Einladung angenommen habe.“92 Am selben Tag sprach Winzer mit seinem designierten Amtskollegen Abdus Samad Azad,

88 Vermerk von Fischer, 8. Januar 1972, PA AA, M 1, C 787/75.

89 Ebd.

90 Dies entsprach auch der Argumentation des Leiters der Bangladesch-Mission in Neu-Delhi zwei Wochen zuvor, s. Diehl an AA, 31. Dezember 1971, PA AA, B 37, Bd. 637.

91 S. handschriftliche „Hinweise für Gen. Minister Winzer“ von Kawretzke und Pierschel, 14. Januar 1972, PA AA, M 1, C 1046/77.

92 Behrends an AA, 9. Januar 1972, PA AA, B 37, Bd. 666. 
um ihm die Bereitschaft der DDR mitzuteilen, Bangladesch offiziell anzuerkennen. Azad bedankte sich bei Winzer für die von Anfang an bewiesene Solidarität der DDR mit dem ostbengalischen Befreiungskampf. „Die DDR ist in Bangla Desh zu einem Begriff geworden“, versicherte er dem ostdeutschen Außenminister und erklärte, dass die Regierung von Bangladesch zur sofortigen Entsendung eines Botschafters in die DDR bereit sei. ${ }^{93}$

Da die verschiedenen DDR-Aktionen zuletzt ein überaus positives Echo in der indischen Presse hervorgerufen hatten, ${ }^{94}$ wollte die Bundesregierung dem Eintreffen Otto Winzers in Neu-Delhi nun mit eigenen, ähnlich öffentlichkeitswirksamen Maßnahmen begegnen. Am Tag der Ankunft Winzers in Neu-Delhi wurde Botschafter Diehl von der Bonner AA-Zentrale angewiesen, den am nächsten Morgen ebenfalls dort erwarteten Mujibur Rahman am Flughafen zu begrüßen, um ihm Grüße der Bundesregierung und die besten Wünsche insbesondere von Bundeskanzler Willy Brandt auszurichten. Das Generalkonsulat in Dhaka wiederum beauftragte man, die Regierung von Bangladesch auf diese westdeutsche Solidaritätsbekundung aufmerksam zu machen und am dortigen Empfang für Mujibur Rahman ebenfalls teilzunehmen - „wenn dies auch die dortigen Vertreter Großbritanniens und Frankreichs tun. “95 Wie erhofft zeigte sich der soeben erst aus der pakistanischen Haft entlassene designierte Premierminister Bangladeschs ausgesprochen dankbar für diese bundesrepublikanische Geste. ${ }^{96}$

Aus deutschlandpolitischer Sicht bedeutete der Umgang mit Mujibur Rahman für die Bundesregierung indes auch ein gewisses Dilemma, galt es doch gegenüber ihm und dem pakistanischen Regierungschef Zulfikar Ali Bhutto, „wegen unserer eigenen Teilungsprobleme mögliche Mißverständnisse [zu] vermeiden“, wie Bundesaußenminister Walter Scheel zu bedenken gab. ${ }^{97}$ Um nicht den Eindruck zu erwecken, die westdeutsche Solidarität mit Rahman bedeute eine indirekte Freigabe der DDR-Anerkennung, griffen bundesrepublikanische Diplo-

93 „Vermerk über ein Gespräch des Außenministers der DDR, Gen. Otto Winzer, mit dem Außenminister der Volksrepublik Bangla Desh, Abdul Samad Azad, am 9. Januar 1972, 13.00 Uhr“ von Radde, undatiert, PA AA, M 1, C 787/75.

94 Diehl an AA sowie Botschaften Rawalpindi, Moskau, Paris und London, 7. Januar 1972, PA AA, B 37, Bd. 694.

95 Van Well an Botschaft Neu-Delhi, Generalkonsulat Dhaka, Botschaften Rawalpindi, London, Paris, Washington und Moskau sowie an SV bei der UNO in New York und bei der NATO in Brüssel, 9. Januar 1972, PA AA, ISLA, Bd. 8133.

96 Behrends an AA, das Generalkonsulat Dacca, die SV bei der UNO in New York sowie die Botschaften Rawalpindi, London, Paris, Washington, Moskau und die SV bei der NATO in Brüssel, 10. Januar 1972, PA AA, B 37, Bd. 694.

97 Sanne an Schilling, 9. Januar 1972, BArch, B 136/6234. 
maten gegenüber Vertretern Bangladeschs neben öffentlich sichtbaren Zeichen der Verbundenheit auch auf diskretere Mittel zurück, um die DDR-Anerkennung durch Bangladesch doch noch zu verhindern.

In der Nacht vor Rahmans Eintreffen in Neu-Delhi stellte der westdeutsche Gesandte Wolfgang Behrends den designierten Außenminister von Bangladesch Abdus Samad Azad zur Rede. Auf die Frage, ob er den DDR-Außenminister tatsächlich nach Bangladesch eingeladen habe, wich Azad aus: ,Winzer habe ihn zu einem Besuch der DDR eingeladen; er habe sich verpflichtet gefühlt, diese Einladung zu erwidern. “ Behrends gab sich daraufhin geschlagen. Die Chancen, eine Anerkennung der DDR durch Bangladesch noch abzuwenden, schätzte er nun deutlich schlechter ein: „Es wird der außenpolitisch unerfahrenen Bangla Desh Regierung sehr schwerfallen, dem Drängen Winzers auf gegenseitige Anerkennung zu widerstehen. “98

Immerhin verfehlte die Begrüßung des Awami-League-Führers durch westdeutsche Diplomaten auf dem Flughafen in Neu-Delhi am Morgen des 10. Januar 1972 ihre Wirkung nicht, wie Behrends später zufrieden berichtete: „Sheikh Mujibur Rahman verabschiedete sich mit besonderer Herzlichkeit von mir. DDRGeneralkonsul Fischer, der bei der Ankunft mein Gespräch mit Mujibur Rahman mitgehört hatte, übermittelte beim Abflug dem Sheikh die besten Wünsche der Regierung und des Volkes der DDR, was von diesem ohne besonderes Interesse und einem kurzen ,thank you' entgegengenommen wurde. “99

Der zeitgleich ebenfalls in Neu-Delhi anwesende, aber nicht zum Empfang Mujibur Rahmans am Flughafen geladene DDR-Außenminister Otto Winzer beschloss daraufhin, schnell Tatsachen zu schaffen. Am 11. Januar 1972 empfing er den Leiter der Bangladesch-Mission im DDR-Generalkonsulat in Neu-Delhi, um ihm im Auftrag von Walter Ulbricht und Willi Stoph ein Schreiben an Mujibur Rahman zu übergeben, in dem die DDR offiziell die völkerrechtliche Anerkennung Bangladeschs aussprach. ${ }^{100}$ Damit hatte die DDR-Führung ihren wichtigsten Trumpf im Umgang mit Bangladesch aus der Hand gegeben und konnte nur noch hoffen, dass die Regierung von Bangladesch $\mathrm{zu}$ ihrem gegebenen Wort stehen würde.

98 Behrends an AA, das Generalkonsulat Dacca, die SV bei der UNO in New York sowie die Botschaften Rawalpindi, London, Paris, Washington, Moskau und die SV bei der NATO in Brüssel, 10. Januar 1972, PA AA, B 37, Bd. 694.

99 Ebd.

100 „Bericht über die Reise des Ministers für Auswärtige Angelegenheiten der DDR nach Indien und Bangladesh vom 8. 1. - 19. 1. 1972“"von Bräsel, 2. März 1972, PA AA, M 1, C 1753/76; zu dessen Wortlaut s. Otto Winzer an Sheik Mujibur Rahman, 10. Januar 1972, PA AA, M 1, C 1045/77. 
Anders als in den westdeutschen Auslandsvertretungen vor Ort, hielt man dies in der Bonner AA-Zentrale für wenig wahrscheinlich und wertete die einseitige Anerkennung Bangladeschs durch die DDR vielmehr als ein Zeichen, dass die Ost-Berliner DDR-Führung ihr „Ziel der unmittelbaren gegenseitigen Anerkennung mit ,Bangla Desh' nicht erreicht“ hatte. Entsprechend sahen westdeutsche Diplomaten zumindest öffentlich auch keine unmittelbare Gefahr einer DDR-Anerkennung durch Indien mehr. Hinsichtlich einer eigenen Anerkennung Bangladeschs gab man allerdings weiterhin zu bedenken, dass Zurückhaltung auch deshalb geboten sei, „um nicht wegen Anerkennung eines geteilten Landes Fehlinterpretationen in der Deutschlandfrage ausgesetzt zu sein."101

Gegenüber seinen indischen Gastgebern bemühte Otto Winzer sich nun, aus der einseitig ausgesprochenen Anerkennung Bangladeschs politisches Kapital zu schlagen. Am selben Tag stellte er das Ergebnis des Bangladeschkriegs in einem Gespräch mit seinem indischen Amtskollegen Swaran Singh als einen gemeinsamen Sieg Indiens und der sozialistischen Staaten (und damit auch der DDR) dar. Er betonte, dass die DDR die Reaktion verschiedener mit Pakistan befreundeter arabischer Staaten auf die nunmehr ausgesprochene Anerkennung Bangladeschs fürchte. Der DDR-Außenminister forderte von Swaran Singh deshalb internationale Rückendeckung: „Der beste Schritt wäre nach Meinung der DDR die Herstellung diplomatischer Beziehungen zwischen der DDR und Indien." Er verlangte eine definitive Zusage sowie einen Zeitpunkt, zu welchem dies geschehen sollte. ${ }^{102}$

Zwar stimmte der indische Außenminister seinem Gast insofern zu, als die DDR „der ,unsichtbare Partner' im Kampf des Volkes von Bangla Desh gewesen“ und die Entscheidung über die Anerkennung der DDR in der Sache bereits getroffen sei. Er ließ sich allerdings weiterhin nicht auf einen konkreten Termin festlegen. Indien habe eine schwierige Zeit hinter sich, erläuterte er, und man wolle „vermeiden, Schwierigkeiten an mehreren Fronten zu schaffen. “103 Damit waren offensichtlich indische Bedenken angesichts möglicher westdeutscher Reaktionen auf eine Anerkennung der DDR gemeint. Noch immer konnte der DDRAußenminister in Indien nicht den erhofften Durchbruch erzielen.

Auch die Vertreter Bangladeschs versuchten die entstandene Situation auf ihre Weise zu nutzen. Noch am selben Tag informierte der Leiter der Bangladesch-Mission Choudhury die bundesrepublikanische Botschaft in Neu-Delhi

101 Sprechzettel zur Lage in Südasien von Berendonck, 11. Januar 1972, PA AA, B 30, Bd. 671. 102 „Vermerk über ein Gespräch des Außenministers, Gen. Otto Winzer, mit dem Außenminister Indiens, Herrn Swaran Singh“ von Radde, 11. Januar, PA AA, M 1, C 787/75.

103 Ebd. 
über die durch Winzer im Namen der DDR ausgesprochene diplomatische Anerkennung von Bangladesch. Er betonte, dass Bangladesch dem Drängen der DDR auf Gegenseitigkeit der Anerkennung nur noch schwer widerstehen könne und appellierte an die Bundesregierung, Bangladesch ihrerseits noch vor Eintreffen Winzers in Dhaka anzuerkennen. Zuletzt argumentierte er auch mit der Logik des Kalten Krieges: Indem der Westen mit der Anerkennung Bangladeschs zögere, werde der jüngste südasiatische Staat ,in die Arme des Ostblocks getrieben [...] und die linksextremen Kräfte“ im Land gestärkt. ${ }^{104}$ Die Bundesregierung zeigte sich hiervon allerdings kaum beeindruckt und betonte stattdessen öffentlich, dass sie ihre Entscheidung in der Bangladesch-Frage unabhängig von der Anerkennung Bangladeschs durch die DDR treffen würde. ${ }^{105}$

Nachdem Otto Winzers Besuch in Dhaka zwischenzeitlich vom 12. auf den 15. Januar 1972 verschoben worden war, ${ }^{106}$ traf er sich am 12. Januar 1972 in Neu-Delhi zu einem Gespräch mit Indira Gandhi. Der DDR-Außenminister kam rasch zur Sache. Als Winzer sie dazu aufforderte, ihm zumindest bei seiner bevorstehenden Rückkehr aus Dhaka wenige Tage später eine konkrete Antwort auf die Frage zu geben, wann die DDR mit der offiziellen Anerkennung durch Indien rechnen könne, wich die indische Premierministerin aus. Sie erläuterte ihm, dass sie aufgrund bevorstehender Wahlen in mehreren indischen Bundesstaaten derzeit nicht mit allen Kabinettsmitgliedern über diese Frage sprechen könne, versprach aber, sich um eine baldige Entscheidung zu bemühen. DDR-Diplomaten nahmen daraufhin an, dass einige der engsten Berater Indira Gandhis sie noch von einer positiven Entscheidung abhalten könnten. ${ }^{107}$

Wenigstens in Bangladesch sollte nun ein möglichst schneller Erfolg erzielt werden. Anlässlich der Vereidigung der neuen Regierung in Dhaka am 12. Januar 1972 wurden Mujibur Rahman und der designierte Außenminister des jungen Staates von den bereits anwesenden DDR-Diplomaten geradezu „belagert“, wie man aus dem westdeutschen Generalkonsulat in Dhaka berichtete. ${ }^{108} \mathrm{Am}$

104 Diehl an AA, Botschaften Luxemburg, Washington, London, Paris, Moskau und das Generalkonsulat Dacca, 11. Januar 1972, PA AA, B 37, Bd. 694.

105 „Pressekonferenz 5/72 am Mittwoch, 12. Januar 1972, 16.00 Uhr, Pressehaus X“, PA AA, B 37, Bd. 694.

106 „Vermerk über ein Gespräch des Außenministers der DDR, Gen. Otto Winzer, mit dem Außenminister der Volksrepublik Bangla Desh, Abdul Samad Azad, am 9. Januar 1972, 13.00 Uhr“ von Radde, undatiert, PA AA, M 1, C 787/75.

107 „Vermerk über ein Gespräch des Außenministers, Gen. Otto Winzer, mit dem Premierminister Indiens, Frau Indira Gandhi, am 12.1.1972“ von Radde, 12. Januar, PA AA, M 1, C 787/75.

108 Kriegler an AA, 13. Januar 1972, BArch, B 136/6234. 
15. Januar 1972 traf dann als erster ausländischer Außenminister überhaupt ${ }^{109}$ auch der DDR-Außenminister selbst in Begleitung unter anderem des DDR-Generalkonsuls Herbert Fischer in Dhaka ein. Er sprach mit dem Staatspräsidenten Bangladeschs Abu Sayeed Chowdhury, Premierminister Mujibur Rahman und Außenminister Abdus Samad Azad, wobei er die Solidarität der DDR mit dem bengalischen Freiheitskampf und die Anerkennung Bangladeschs durch die DDR besonders hervorhob. ${ }^{110}$ Im MfAA war man Bangladesch gegenüber zu großen Zugeständnissen bereit, um die zuvor zugesagte Gegenseitigkeit der Anerkennung zu sichern. Man würde sogar „die Kosten für die Einrichtung und den Unterhalt der Botschaft der Volksrepublik Bangla Desh in Berlin [...] übernehmen“111, was deutlich über die Handlungsempfehlungen der Sowjetunion hinausging. ${ }^{112}$

Am Tag seiner Ankunft in Dhaka führte Otto Winzer dann ein längeres Gespräch mit seinem Amtskollegen Abdus Samad Azad. Dieser schlug dem DDRAußenminister ein gemeinsames Kommuniqué vor, in dem die Aufnahme diplomatischer Beziehungen zwischen beiden Staaten verkündet werden sollte. Dabei wies er darauf hin, dass westdeutsche Diplomaten zwischenzeitlich gegen diesen beabsichtigten Schritt zu intervenieren versucht hätten und Indira Gandhi ihn über die Bangladesch-freundliche Haltung des westdeutschen Bundeskanzlers Willy Brandt informiert hätte. Winzer zeigte sich sehr dankbar, dass die Regierung Bangladeschs dennoch $\mathrm{zu}$ ihrem Wort stehen wollte und machte einige inhaltliche Vorschläge zum Kommuniqué. ${ }^{113}$ Vor allem betraf dies die Formulierung, beide Regierungen seien „übereingekommen, diplomatische Beziehungen zwischen beiden Staaten entsprechend der Wiener Konvention vom 18. April 1961 herzustellen“, ${ }^{114}$ wodurch die staatliche Souveränität der DDR zusätzlich betont werden sollte.

In einem Gespräch mit Mujibur Rahman bedankte dieser sich am selben Tag bei Winzer für die Anerkennung Bangladeschs durch die DDR, stimmte der Herstellung diplomatischer Beziehungen zwischen den beiden Ländern zu und

109 Schneider: Die Außenpolitik der DDR gegenüber Südasien, S. 20; 23.

110 Enders an AA und Botschaft Rawalpindi, 22. Januar 1972, PA AA, ISLA, Bd. 8133.

111 „Hinweise für das Gespräch mit dem Ministerpräsidenten von Bangla Desh, Sheik Mujibur Rahman und dem Außenminister von Bangla Desh“, 13. Januar 1972, PA AA, M 1, C 1046/77.

112 Vgl. handschriftliche „Hinweise für Gen. Minister Winzer“ von Kawretzke und Pierschel, 14. Januar 1972, PA AA, M 1, C 1046/77.

113 „Vermerk über die Gespräche mit Außenminister Abdus Samad Azad am 15.1.1972“, 17. Januar, PA AA, M 1, C 787/75.

114 „Gemeinsames Kommuniqué über den Besuch des Ministers für Auswärtige Angelegenheiten der Deutschen Demokratischen Republik, Otto Winter, in der Volksrepublik Bangladesh“, undatiert, PA AA, M 1, C 1046/77. 
bestätigte am Folgetag das ausgearbeitete gemeinsame Kommuniqué „ohne Einschränkung. “115 Otto Winzer wiederum bemühte sich bei diesem ersten Treffen sofort darum, Mujibur Rahman die Ost-Berliner Sicht auf das deutsch-deutsche Verhältnis näherzubringen. Er betonte, „daß es genauso wie es zwischen Bangladesh und Pakistan kein sogenanntes innerpakistanisches Sonderverhältnis geben kann, auch zwischen der sozialistischen DDR und der imperialistischen BRD kein sogenanntes innerdeutsches Sonderverhältnis geben wird. “116

Nach Indien und Bhutan wurde die DDR mit Veröffentlichung des Kommuniqués am 16. Januar 1972 das dritte Land, das Bangladesch anerkannte, und der erste europäische Staat überhaupt. ${ }^{117}$ Den „Wettlauf“ um Bangladesch hatte die DDR damit klar gewonnen. ${ }^{118}$

\section{Deutsch-indische Regierungskonsultationen}

Wichtiger als die Anerkennung der DDR durch Bangladesch war aus westdeutscher Sicht nach wie vor die Frage, welche Reaktionen dieser Schritt in der indischen Regierung hervorrufen würde. ${ }^{119}$ Diese sandte in diesen kritischen Tagen ausgesprochen widersprüchliche Signale: Einerseits nährte der indische Botschafter in Bonn die dortigen Sorgen, als er am 12. Januar 1972 - am Tag nach Winzers Verkündigung der Anerkennung Bangladeschs durch die DDR - im Bundeskanzleramt vorsprach, um unter anderem zu erfragen, in welchen Punkten bei ihren bilateralen Verhandlungen denn überhaupt noch größere Meinungsverschiedenheiten zwischen Bundesrepublik und DDR bestünden. ${ }^{120}$

Die Bundesregierung musste daraufhin annehmen, dass der indische Botschafter im Auftrag seiner Regierung auszuloten versuchte, wie man im Bundes-

115 „Vermerk über Gespräch mit Premierminister, Sheikh Mujibur Rahman am 15.1.72, 17.15 Uhr bis 17.55 Uhr in dessen offizieller Residenz“ von Herbert Fischer, 17. Januar, PA AA, M 1, C 787/75. 116 „Bericht über die Reise des Ministers für Auswärtige Angelegenheiten der DDR nach Indien und Bangladesh vom 8. 1. - 19. 1. 1972“ von Bräsel, 2. März 1972, PA AA, M 1, C 1753/76.

117 Schneider: Die Außenpolitik der DDR gegenüber Südasien, S. 3; 20; Heß: Bangladesch. Tragödie einer Staatsgründung, S. 139; Voigt: Die Indienpolitik der DDR, S. 661.

118 S. hierzu auch „Niederschrift über die Unterredung zwischen Genossen Winzer und Genossen Gromyko am 19. Januar 1972“, 20. Januar 1972, BArch, DY 30/11654.

119 Die Bonner AA-Zentrale empfahl vor diesem Hintergrund, dem indischen Drängen auf weitere Kapitalhilfezahlungen nur gestaffelt nachzugeben, da dies eine „bremsende Wirkung“ auf die unerwünschte DDR-Anerkennung haben würde, s. Vermerk von Herbst, 6. Januar 1972, PA AA, B 30, Bd. 671.

120 Vermerk von Sahm, 12. Januar 1972, PA AA, B 37, Bd. 666. 
kanzleramt wohl auf eine Anerkennung der DDR reagieren würde. Andererseits wiederum versicherte derselbe Botschafter Kewal Singh dem westdeutschen AAStaatssekretär Paul Frank am Folgetag, der Besuch des DDR-Außenministers Otto Winzer in Neu-Delhi habe die indische Haltung in der Deutschlandfrage keinesfalls geändert. Frank sagte ihm daraufhin die schnelle und unbürokratische Hilfe der Bundesregierung beim Wiederaufbau Ostbengalens, nicht aber konkret die von der indischen Regierung erhoffte Anerkennung Bangladeschs zu. ${ }^{121}$

Botschafter Diehl in Neu-Delhi jedenfalls sah Mitte Januar 1972 nur noch geringe Chancen, „die Anerkennung der DDR durch Indien wenigstens noch bis Mai 1972 hinauszuzögern“, wie das MfS später in Erfahrung brachte. Er ergriff nun also selbst die Initiative: Am 11. Januar 1972 deutete er einem indischen Regierungsvertreter gegenüber an, die Bundesregierung würde die diplomatischen Beziehungen zu Indien abbrechen, falls die Regierung Indira Gandhi sich dazu entschließen sollte, die DDR anzuerkennen. Sein Gesprächspartner zeigte sich von dieser Drohung durchaus beeindruckt und gab zu verstehen, dass seine Regierung sich eine solch harsche Konsequenz derzeit nicht leisten konnte. ${ }^{122}$

Nach seiner Rückkehr aus Dhaka musste der DDR-Außenminister Otto Winzer am 16. Januar 1972 in Neu-Delhi erfahren, dass die indische Regierung sich von seinem Vorpreschen in der Causa „Bangladesch“ nicht so leicht beeindrucken ließ wie erhofft. Sein indischer Amtskollege Swaran Singh erläuterte ihm, dass seine Regierung die Neue Ostpolitik der Regierung Brandt sehr schätze und ihr dabei keine Schwierigkeiten bereiten wolle. Die Einwände Singhs gegen eine sofortige Anerkennung der DDR provozierten Winzer zur verzweifelten Erwiderung: „Ebenso wie es keine innerpakistanischen Beziehungen geben kann, kann es keine innerdeutschen Beziehungen zwischen der sozialistischen DDR und der kapitalistischen BRD geben. “ Dennoch ließ sich der indische Außenminister weiterhin nicht auf ein konkretes Datum einer DDR-Anerkennung festlegen. Er versprach lediglich, dass DDR-Generalkonsul Herbert Fischer noch während seiner Amtszeit offiziell zum ersten ordentlichen Botschafter der DDR in Indien ernannt werden würde. ${ }^{123}$

Zurück in Europa konnte Winzer seine Enttäuschung kaum verbergen. In einem Bericht an die politische Führung der DDR schrieb er nach dem Treffen mit dem indischen Außenminister, das Gespräch habe gezeigt, „daß [die] indi-

121 Berendonck an Botschaft Neu-Delhi, 13. Januar 1972, PA AA, B 37, Bd. 666.

122 „Information über Tendenzen in der indischen Außenpolitik gegenüber imperialistischen Hauptmächten, Pakistan und der DDR“, 18. Januar 1972, BStU, MfS, HV A, Nr. 379.

123 „Vermerk über 2. Gespräch mit Außenminister Swaran Singh am 17.1.72“ von Herbert Fischer, undatiert, PA AA, M 1, C 787/75. 
sche Regierung nach wie vor Illusionen über [die] BRD-Politik hat. “124 In der DDRVertretung in Neu-Delhi rechnete man nach positiven Reaktionen im indischen Außenministerium auf Winzers Besuch dennoch mit einer indischen DDR-Anerkennung um den 15. März 1972. ${ }^{125}$

Auch im westdeutschen Auswärtigen Amt wurde das beständige Hin und Her der indischen Regierung in dieser Frage mit Sorge beobachtet. Man war dort allerdings kaum dazu bereit, sich dieser Willkür zu fügen, sondern wollte weiter Einfluss im eigenen Sinne nehmen. Die Strategie, auf die die Bundesregierung Mitte Januar 1972 verfiel, war bestechend einfach: Am 20. Januar 1972 würde AA-Staatssekretär Paul Frank ohnehin zu deutsch-indischen Regierungskonsultationen in Neu-Delhi eintreffen. Bei der Gelegenheit sollte er seinen indischen Gesprächspartnern im Gegenzug zu einem weiteren Aufschub der DDR-Anerkennung eine Anerkennung wiederum des indischen Protegés Bangladesch durch die BRD sowie durch die anderen fünf Staaten der Europäischen Wirtschaftsgemeinschaft (EWG) und Großbritannien anbieten.

Bereits Anfang Januar 1972 hatten luxemburgische Diplomaten der Bundesregierung Konsultationen über ein gemeinsames Auftreten der EWG-Staaten (Belgien, Niederlande, Luxemburg, Frankreich, Italien und die Bundesrepublik Deutschland) sowie Großbritanniens gegenüber Bangladesch vorgeschlagen. ${ }^{126}$ Vor allem die britische Regierung befürwortete eine rasche Anerkennung Bangladeschs, um Mujibur Rahman international den Rücken zu stärken, ${ }^{127}$ war dabei aber auch nicht unbeeindruckt von der Drohung Bhuttos, Pakistan werde im Falle einer Anerkennung Bangladeschs durch ein Commonwealth-Mitglied selbst aus diesem britisch geführten Staatenbündnis austreten. ${ }^{128}$

Die Bereitschaft zur von der indischen Regierung angestrebten internationalen Anerkennung Bangladeschs war bei den westeuropäischen Partnern der Bundesregierung prinzipiell bereits vorhanden. Am 14. Januar 1972 erhielten die Bonner Diplomaten schließlich deren Zusage, mit der Anerkennung von Bangladesch wenigstens noch bis nach den deutsch-indischen Regierungskonsultationen zu warten. ${ }^{129}$

124 Winzer an Honecker, Stoph, Axen und Florin, 18. Januar 1972, BArch, DY 30/98813.

125 Fischer an Winzer und Radde, 20. Januar 1972, BArch, DY 30/98813.

126 Vermerk von Berendonck, 5. Januar 1972, PA AA, B 30, Bd. 654.

127 Edward Heath an Willy Brandt, 15. Januar 1972, BArch, B 136/6234.

128 Diehl an AA, 17. Januar 1972, BArch, B 136/6234. Aus Protest gegen die Anerkennung Bangladeschs durch Australien und Neuseeland machte Bhutto diese Drohung später tatsächlich wahr, s. Sprechzettel zur „Lage in Südostasien“, 2. Februar 1972, PA AA, B 37, Bd. 692.

129 Vermerk über „Konsultation der Sechs und Großbritanniens über die Frage der Anerkennung Bangla Deshs“, 3. Februar 1972, PA AA, B 37, Bd. 692; vgl. Müller an Botschaften Neu-Delhi, 
Es war dann tatsächlich vor allem diese Aussicht auf eine gemeinsame Anerkennung Bangladeschs durch die EWG-Staaten und Großbritannien, die sich als das entscheidende Druckmittel in den Händen der Bundesregierung herausstellen sollte. Nach dem vernichtenden Sieg gegen Pakistan suchte die indische Regierung den Weg zurück zu internationalem Gleichgewicht und regionaler Stabilität. Das Verhältnis zur US-Regierung war in diesem Konflikt schwer beschädigt worden und so gewannen die westeuropäischen Staaten als Ansprechpartner aus indischer Sicht plötzlich stark an Attraktivität. ${ }^{130}$ Außerdem war die Regierung Indira Gandhi sehr daran interessiert, Bangladesch möglichst rasch den Weg in die internationale Staatengemeinschaft (und damit endgültig weg von Westpakistan) zu ebnen. ${ }^{131}$

Für die Bundesregierung galt es allerdings auch, etwaige pakistanische Reaktionen auf die dort ausdrücklich unerwünschte Anerkennung Bangladeschs zu bedenken. Nachdem diese Bangladesch anerkannten, hatte das pakistanische Außenministerium seine diplomatischen Beziehungen zur Mongolei und zu Polen abgebrochen, ${ }^{132}$ während aus Burma und Nepal daraufhin zumindest die pakistanischen Botschafter zurückberufen wurden. ${ }^{133}$ Obgleich Bhutto öffentlich beteuerte, diese Maßnahmen seien keineswegs Ausdruck einer pakistanischen Hallstein-Doktrin gegenüber Bangladesch, ${ }^{134}$ stand aus Bonner Sicht durchaus $\mathrm{zu}$ befürchten, dass die pakistanische Regierung die voreilige Anerkennung seines abtrünnigen östlichen Landesteils durch die BRD mit einer Anerkennung wiederum der DDR abstrafen würde. ${ }^{135}$ Damit hätte der Versuch, die indische Regierung durch eine Anerkennung Bangladeschs von einer Anerkennung der DDR abzuhalten, in Pakistan genau den gegenteiligen Effekt.

Bis zur Abreise von Staatssekretär Paul Frank nach Indien war die Bundesregierung nach außen hin freilich sehr darum bemüht, die Bedeutung der DDR-

London, Rawalpindi, Paris, Rom, Brüssel, Den Haag, Luxemburg und Washington, 14. Januar 1972, PA AA, ISLA, Bd. 8133.

130 AA / IfZ: $A A P d B, 1972$, Bd. 1, Dok. 14. Ähnliche Motive unterstellten westdeutsche Diplomaten vor Ort in dieser Zeit auch dem jungen Bangladesch, s. Kriegler an AA und Botschaft Rawalpindi, 13. Januar 1972, PA AA, ISLA, Bd. 8133.

131 AA / IfZ: $A A P d B, 1972$, Bd. 1, Dok. 14, Anm. 8. Auch während der deutsch-indischen Regierungkonsultationen argumentierte die indische Seite später, eine internationale Anerkennung Bangladeschs sei förderlich für seine Stabilität, s. Diehl an AA, 25. Januar 1972, PA AA, B 30, Bd. 654.

132 Sachstand „Frage der Anerkennung von ,Bangla Desh’“, Referat I B 5 an Büro Staatssekretäre, 18. Januar 1972, PA AA, B 37, Bd. 666.

133 Diehl an AA, 23. Januar 1972, PA AA, B 37, Bd. 666.

134 Hendus an AA, 26. Januar 1972, PA AA, B 37, Bd. 693.

$135 \mathrm{Zu}$ dieser Interessenabwägung, s. Berger an AA, 17. Januar 1972, BArch, B 136/6234. 
Anerkennung durch Bangladesch herunterzuspielen. In einer Pressekonferenz am 12. Januar 1972 hieß es, der Besuch Franks in Neu-Delhi habe keineswegs als einziges Ziel, eine indische DDR-Anerkennung $\mathrm{zu}$ verhindern. ${ }^{136}$ Und auch der bundesrepublikanische Botschafter Diehl versuchte öffentlich zu beschwichtigen, die indische Regierung werde sich weder durch den leicht durchschaubaren Schachzug der DDR locken noch durch die Sowjetunion zu einer DDR-Anerkennung drängen lassen, solange sie diesen Schritt nicht selbst zu gehen wünsche. ${ }^{137}$

Bei den deutsch-indischen Regierungskonsultationen selbst zeigte sich eine gute Woche später dann allerdings ein etwas anderes Bild. Bereits zu Beginn der Gespräche Paul Franks mit seinem indischen Amtskollegen T. N. Kaul am 20. Januar 1972, machte jener deutlich, dass eine Anerkennung Bangladeschs durch gleich mehrere europäische Staaten tatsächlich sehr im indischen Interesse wäre. Ein solcher Schritt würde es seiner Regierung außerdem erleichtern, der Bundesregierung „durch weitere Zurückhaltung in der Frage indischer DDR-Anerkennung entgegenzukommen. “138 Dieser indische Vorschlag entsprach genau den Vorstellungen, mit denen Staatssekretär Frank nach Indien gereist war, sodass er beherzt darauf einging: Es sei sogar möglich, dass die Entscheidung über eine Anerkennung Bangladeschs durch die Bundesregierung noch vor Ende der bilateralen Konsultationen falle. ${ }^{139}$

Daraufhin erhöhte der indische Staatssekretär Kaul den Druck auf seinen westdeutschen Gesprächspartner. Er teilte ihm mit, bei aller Rücksichtnahme auf die bundesrepublikanische Entspannungspolitik könne die indische Regierung „die Anerkennung der bestehenden Realitäten [in Deutschland] nicht mehr sehr viel länger hinauszögern.“ Er verwies darauf, dass die DDR versucht habe, „mit der Bangla Desh Anerkennung ein Geschäft mit Indien zu machen.“ Hierauf sei seine Regierung aber nicht eingegangen. ${ }^{140}$ Die DDR hätte sich durch ihre Solidarität im Bangladeschkrieg und mit der Anerkennung von Bangladesch allerdings große Sympathien in der indischen Öffentlichkeit und im Parlament erworben. ${ }^{141}$ Zwar wünsche man die bilateralen Gespräche zwischen Bundesrepublik und

136 Huber an Generalkonsulat Dacca, 13. Januar 1972, PA AA, DHAK, Bd. 9092.

137 „Hans-Joachim Werbke interviewt BRD-Botschafter in Neu-Delhi, Günter Diehl“, 17. Januar 1972, BArch, DY 30/98813.

138 AA / IfZ: $A A P d B, 1972$, Bd. 1, Dok. 14.

139 „Indo-F.R.G. Talks“, 20. Januar 1972, NAI, Ministry of External Affairs, H I/121(5)/72.

140 Frank/Diehl an AA, 20. Januar 1972, PA AA, B 37, Bd. 666. Zuvor hatte er das Vorgehen Winzers gar als „Erpressung“ bezeichnet, s. Gisela Bonn an Willy Brandt, 17. Januar 1972, BArch, B 136/6234.

141 Frank/Diehl an AA, 20. Januar 1972, PA AA, B 37, Bd. 666. 
DDR natürlich keinesfalls zu stören, dennoch bedeute dies eine gewisse Verpflichtung. ${ }^{142}$

Hierauf begann ein regelrechtes Kräftemessen der beiden Spitzendiplomaten: Staatssekretär Frank wies Kaul darauf hin, dass die Anerkennung der DDR durch Chile sich zuletzt durchaus negativ auf die bilateralen Beziehungen zwischen der BRD und Chile ausgewirkt habe. Kaul entgegnete daraufhin, die indische Regierung erwarte allerdings auch eine Berücksichtigung ihrer eigenen Interessen. An dieser Stelle warf Paul Frank ein, „,wenn er zynisch wäre, würde er der indischen Seite ein Tauschgeschäft anbieten.“143

Obgleich er es nicht offen aussprach, bot Frank seinem indischen Gesprächspartner damit eine Anerkennung Bangladeschs durch die BRD im Gegenzug zu einem weiteren Aufschub der indischen DDR-Anerkennung an. Auf dieses beinahe ausgesprochene Angebot ging Kaul nun ein, indem er Frank bestätigte, die angesprochene „Anerkennung Bangla Deschs durch die Bundesregierung würde helfen, den Druck zugunsten einer indischen DDR-Anerkennung zu verringern. " ${ }^{144}$ In einer abschließenden gemeinsamen Sitzung wies Kaul noch einmal darauf hin, „daß es Indien dabei auf Tage ankomme“, aber auch, dass „die indische Regierung nichts tun werde, was den Erfolg der Entspannungsbemühungen der Bundesregierung erschweren würde. “ ${ }^{145}$

Später betonte Staatssekretär Paul Frank zwar, dass es bei seinem Besuch in Indien keineswegs darum gegangen sei, „eine Feuerwehr nach New Delhi zu entsenden, die das wieder löschen soll, was angeblich Herr Winzer dort angerichtet hat. " ${ }^{146}$ Seine Unterredung mit T. N. Kaul hatte jedoch genau den bestrittenen Effekt. Auch wenn es ihm nicht gelungen war, seine indischen Gesprächspartner zusätzlich zu der Zusage zu bewegen, die Anerkennung der DDR vom Beitritt der beiden deutschen Staaten zur UNO abhängig zu machen, ${ }^{147}$ war Paul Frank mit seinem Besuch in Neu-Delhi später sehr zufrieden. Den Umstand, dass ihre europäischen Partner dem Wunsch der Bundesregierung nachgekommen waren, von einer Anerkennung Bangladeschs bis zu den deutsch-indischen Konsultationen abzusehen, bezeichnete er im Nachhinein als „einen großen taktischen

142 „Indo-F.R.G. Talks“, 20. Januar 1972, NAI, Ministry of External Affairs, H I/121(5)/72.

143 Diehl an AA, 31. Januar 1972, PA AA, B 37, Bd. 666; vgl. auch „Indo-F.R.G. Talks“, 20. Januar 1972, NAI, Ministry of External Affairs, H I/121(5)/72.

144 Diehl an AA, 31. Januar 1972, PA AA, B 37, Bd. 666.

145 AA / IfZ: $A A P d B, 1972$, Bd. 1, Dok. 14, Anm. 8.

146 „Kurzprotokoll 43. Sitzung des Ausschusses für wirtschaftliche Zusammenarbeit am Donnerstag, dem 27. Januar 1972, 9.30 Uhr in Bonn, Bundeshaus“, PA AA, B 37, Bd. 666.

147 AA / IfZ: $A A P d B, 1972$, Bd. 1, Dok. 14. 
Vorteil.“148 Er empfahl der Bundesregierung allerdings auch, Bangladesch nun tatsächlich möglichst bald anzuerkennen. ${ }^{149}$

Die Bundesregierung war zum Zeitpunkt der deutsch-indischen Konsultationen in Neu-Delhi vollauf dazu bereit, als Sanktion einer DDR-Anerkennung die Entwicklungshilfezahlungen an Indien einzustellen und hatte in den drei Wochen vor den Konsultationen bereits den Vollzug bestehender Abkommen blockiert, um den Druck auf Staatssekretär Franks indische Gesprächspartner zu erhöhen. ${ }^{150}$ Ein Druckmittel, das nach dem Dritten Indisch-Pakistanischen Krieg noch wirksamer geworden war, als die indische Regierung um US-amerikanische Entwicklungshilfezahlungen bangen musste. In Indien wiederum war Anfang Januar 1972 „die Anerkennung der DDR praktisch beschlossene Sache“, wie indische Regierungsvertreter später bestätigten. Erst die Konsultationen mit AA-Staatssekretär Frank bewogen die indische Regierung dazu, ihre Entscheidung noch einmal zu überdenken. ${ }^{151}$ Die DDR-Diplomaten hatten ihr Ziel also nur äußerst knapp verfehlt.

\section{Pakistanische Interventionsversuche}

Die öffentlichen Anzeichen einer bevorstehenden Anerkennung Bangladeschs durch die Bundesregierung versetzten das pakistanische Außenministerium in Alarmbereitschaft. Kaum war Staatssekretär Frank aus Indien nach Bonn zurückgekehrt, sprach der dortige pakistanische Botschafter Kharas im AA vor, um die Bundesregierung von der angekündigten Anerkennung Bangladeschs abzuhalten. ${ }^{152}$ Er erinnerte an das frühere Wohlverhalten Pakistans in der Frage der DDRAnerkennung und mahnte, eine Anerkennung Bangladeschs durch die Bundesrepublik stelle aus pakistanischer Sicht „einen unfreundlichen, ja feindlichen Akt dar.“ Im Auswärtigen Amt argumentierte man daraufhin, dass es auch im pakistanischen Interesse sei, ein Abgleiten Indiens und Bangladeschs in die Ein-

148 So konnte Frank seinem indischen Gesprächspartner glaubhaft vermitteln, dass auch die Entscheidung der EWG-Staaten und Großbritanniens über die Bangladesch-Anerkennung vom Erfolg der deutsch-indischen Konsultationen abhing, s. „Kurzprotokoll 43. Sitzung des Ausschusses für wirtschaftliche Zusammenarbeit am Donnerstag, dem 27. Januar 1972, 9.30 Uhr in Bonn, Bundeshaus“, PA AA, B 37, Bd. 666.

149 AA / IfZ: $A A P d B$, 1972, Bd. 1, Dok. 14; ähnlich auch Diehl an AA, 23. Januar 1972, PA AA, B 37, Bd. 666.

150 Pflaumer an Erhard Eppler, 27. Januar 1971, BArch, B 213/6777.

151 Behrends an AA, 26. Mai 1972, PA AA, B 37, Bd. 666.

152 Vermerk von Thönnes, 25. Januar 1972, PA AA, B 30, Bd. 673. 
flusssphäre des sozialistischen Staatenbündnisses zu vermeiden. Im Übrigen, so die unmissverständliche Drohung, wünsche Präsident Bhutto sicherlich nicht, die ohnehin bereits schwierige Lage Pakistans auf dem Subkontinent durch eine scharfe Reaktion gegenüber mehreren wichtigen europäischen Staaten noch weiter $\mathrm{zu}$ erschweren? ${ }^{153}$

An dieser Stelle zeigte sich die zweite Ebene der bundesrepublikanischen Strategie, Bangladesch im Verbund mit weiteren einflussreichen europäischen Staaten anzuerkennen. Die Bundesregierung konnte dadurch nicht nur der indischen Regierung das überaus attraktive Angebot einer Anerkennung ihres jungen Protegés durch gleich mehrere westliche Staaten gleichzeitig unterbreiten, sondern das Handeln im Verbund diente der Bundesregierung auch als Schutz ihrer deutschlandpolitischen Interessen in Pakistan. Denn die Regierung Bhutto würde es sich kaum leisten können, mehrere bedeutende Entwicklungshilfegeber gleichermaßen für die unliebsame Anerkennung Bangladeschs abzustrafen. ${ }^{154}$ Etwaige pakistanische Reaktionen dürften also wohl eher milde ausfallen. ${ }^{155}$

Gegenüber Indien gelang der Coup der Bundesregierung. Am 28. Januar 1972 teilten indische Diplomaten in Bonn mit, Indira Gandhi „beabsichtige nicht, die DDR in absehbarer Zeit anzuerkennen. " ${ }^{156}$ Die indische Gegenfrage allerdings, ob denn auch bereits über eine bundesrepublikanische Anerkennung Bangladeschs entschieden sei, wollte man von deutscher Seite zu diesem Zeitpunkt nicht ganz so freimütig beantworten. ${ }^{157}$ Hintergrund war, dass die westeuropäischen Staaten sich entgegen der westdeutschen Darstellung gegenüber Indien über den Zeitpunkt einer gemeinsamen Anerkennung noch keineswegs einig waren.

Zumindest Frankreich und Italien würden absehbar aus der Gruppe ausscheren. Frankreichs Regierung scheute davor zurück, die arabischen Staaten durch die Anerkennung einer abtrünnigen Provinz eines ihrer islamischen Partnerländer vor den Kopf zu stoßen. In Italien wiederum machte man einen vollständigen Abzug der indischen Truppen aus Ostbengalen zur Voraussetzung für eine Anerkennung von Bangladesch. Auch nach mehrtägigen Diskussionen beharrten Frankreich und Italien weiter auf ihren Positionen, während die britische Regierung eigenständig entschied, Bangladesch am 4. Februar 1972 anzuerkennen, und die Benelux-Länder die Anerkennung zum selben Zeitpunkt ankündigen, aber

153 Müller an Botschaften Rawalpindi und Neu-Delhi, 26. Januar 1972, PA AA, ISLA, Bd. 8133.

154 Sprechzettel zur „Lage in Südostasien“, 2. Februar 1972, PA AA, B 37, Bd. 692; zuvor bereits Diehl an AA, 25. Januar 1972, PA AA, B 30, Bd. 654.

155 Frank an Generalkonsulat Dacca, 26. Januar 1972, PA AA, DHAK, Bd. 9092.

156 AA / IfZ: $A A P d B, 1972$, Bd. 1, Dok. 14, Anm. 5.

157 Vermerk von Müller, 28. Januar 1972, PA AA, B 37, Bd. 666. 
erst in der folgenden Woche vollziehen würden. Die Bundesregierung beschloss, sich Großbritannien anzuschließen und den pakistanischen Präsidenten Bhutto sicherheitshalber vorab über den beabsichtigten Schritt zu informieren. ${ }^{158}$

Auch weitere Vorsprachen des pakistanischen Botschafters bei Bundesaußenminister Walter Scheel ${ }^{159}$ und sein Appell an führende SPD-Funktionäre, die Situation seiner Regierung sei doch durchaus mit derjenigen der Bundesregierung im Umgang mit der DDR vergleichbar, ${ }^{160}$ konnten die Bundesregierung nicht mehr umstimmen. Ebenso wenig die Warnung des westdeutschen Botschafters in Pakistan Norbert Berger, es sei zumindest denkbar, dass die pakistanische Regierung in Reaktion auf die Anerkennung von Bangladesch die diplomatischen Beziehungen zur Bundesrepublik abbrechen könnte. ${ }^{161}$

Das Bonner Kalkül ging dann auch gegenüber Pakistan unerwartet gut auf. Die Anerkennung Bangladeschs durch die Bundesrepublik und Großbritannien am 4. Februar $1972^{162}$ wurde von pakistanischer Seite zwar sehr bedauert. ${ }^{163}$ Eine vom pakistanischen Botschafter Kharas noch am selben Tag angekündigte harsche Reaktion seiner Regierung ${ }^{164}$ blieb aber aus. Erst zwei Wochen später brachte Präsident Bhutto in einem Brief an Willy Brandt seinen Unmut über die bundesrepublikanische Anerkennung Bangladeschs zum Ausdruck: ,we felt that the Federal Republic of Germany, facing a similar situation, would not have been in the forefront of Western European countries granting recognition to ,Bangla Desh'“" ${ }^{165}$ Konsequenzen hatte dieser Unmut für die Bundesregierung aber nicht.

Im indischen Außenministerium wiederum zeigte man sich zutiefst dankbar, dass die Bundesregierung mit der Anerkennung Bangladeschs Wort gehalten hatte. Noch Monate später lobte der indische Botschafter in Bonn Willy Brandt in internen Berichten als den aufrichtigsten Regierungschef Westeuropas. In seiner Begeisterung schien er sogar fast die DDR zu vergessen: „FRG was the first country in Europe to recognise Bangladesh. "166 Damit waren die Ost-Berliner

158 Vermerk über „Konsultation den der Sechs und Großbritanniens über die Frage der Anerkennung Bangla Deshs“, 3. Januar 1972, PA AA, B 37, Bd. 692.

159 Mueller an Generalkonsulat Dacca, 31. Januar 1972, PA AA, DHAK, Bd. 9092.

160 Kharas an Hans-Eberhard Dingels, 28. Januar 1972, PA AA, B 37, Bd. 692.

161 Berger an AA, 2. Februar 1972, PA AA, DHAK, Bd. 9092.

162 AA / IfZ: $A A P d B, 1972$, Bd. 1, Dok. 14, Anm. 8.

163 Botschaft Pakistans in der Bundesrepublik Deutschland an AA, 15. Februar 1972, PA AA, B 37, Bd. 692.

164 Berendonck an Botschaften Rawalpindi und Neu-Delhi, 4. Februar 1972, PA AA, B 37, Bd. 698. 165 Bhutto an Willy Brandt, 23. Februar 1972, PA AA, B 37, Bd. 698.

166 „Meeting of the Heads of Indian Offices in FRG - Minutes of the discussion on the forenoon of 19th June, 1972“, undatiert, NAI, Ministry of External Affairs, H I/1012(49)/72. 
Bemühungen, die Regierung Indira Gandhi durch einseitige Stellungnahme im Bangladeschkrieg auf seine Seite zu ziehen, durch die Bundesregierung erfolgreich neutralisiert worden.

\section{Indien vertröstet die DDR}

Während der westdeutsche AA-Staatssekretär Paul Frank sich später damit brüsten konnte, die indische Regierung bei den Konsultationen im Januar 1972 durch die Kombination eines attraktiven Angebots mit empfindlichen Drohungen erfolgreich von einer Anerkennung der DDR abgehalten zu haben, hielten DDR-freundliche Abgeordnete des indischen Unterhauses ihrem Außenminister Swaran Singh Mitte März 1972 eben diesen Umstand vor:

Is it not a fact that due to pressure exerted by West Germany and its friends through aid, etc., they prevented the Government of India from granting full diplomatic recognition to the German Democratic Republic ${ }^{167}$

Swaran Singh verneinte dies zwar vehement, musste auf weitere kritische Rückfragen seitens der Parlamentarier jedoch auch eingestehen, dass die DDR sich im Bangladeschkrieg äußerst solidarisch mit Indien verhalten hatte. Dieser Umstand spräche sehr dafür, die DDR bald diplomatisch anzuerkennen. ${ }^{168}$

Einige Aktionen des dortigen westdeutschen Botschafters, Günter Diehl, drohten außerdem die Bemühungen der Bundesregierung um Durchsetzung ihrer deutschlandpolitischen Interessen in Indien zu desavouieren. Als Ende Januar 1972 eine indische Unterhausabgeordnete in der indischen Tageszeitung National Herald die Teilung Pakistans mit der deutschen Teilung verglich und die indische Regierung aus diesem Grund zur Anerkennung der DDR aufforderte, antwortete Diehl ihr in einem Leserbrief, der zwei Wochen später ebenfalls im National Herald erschien. Ausführlich stellte er darin die Missstände in der DDR heraus: „The prisons are full of political dissidents in East Germany, not in the Federal Republic. [...] for an East Berliner, it is impossible to see his neighbours a few hundred yards beyond the Wall.” Für Diehl war das Verhältnis der beiden deutschen Staaten also ein ganz anderes als das von Ost- und Westpakistan, „in

167 „Lok Sabha Starred Question No. 48 to be answered on the 16th March, 1972. Diplomatic relations with German Democratc Republic”, 16. März 1972, PA AA, B 37, Bd. 666. Ähnlich äußerte sich zwei Monate später auch Joint Secretary Dr. S. Sinha, vgl. Behrends an AA, 26. Mai 1972, PA AA, B 37, Bd. 666.

168 Ebd. 
Bangla Desh self-determination, democracy and human right[s] meant independence, while in Germany the same principle[s] would mean reunification."

Diese öffentliche Tirade Diehls stieß in der indischen Regierung auf wenig Verständnis. Anfang April 1972 stellte der stellvertretende indische Außenminister, Surendra Pal Singh, vor dem indischen Oberhaus zum Vorgehen des westdeutschen Botschafters klar: „We disapprove as a matter of principle of any propaganda by any foreign mission against third countries with whom India maintains friendly relations." ${ }^{170}$ Während DDR-Diplomaten diese Aussage des indischen Außenpolitikers dazu nutzen konnten, das Verhalten der Bundesregierung in Indien öffentlich anzuprangern, reagierte man in der Bonner AA-Zentrale recht gelassen. Westdeutsche Diplomaten schlugen vor, im Falle künftiger ostdeutscher Angriffe auf die bundesrepublikanische Botschaft in Neu-Delhi auf Singhs Bemerkung zurückzukommen. Im Übrigen betreibe auch die indische Botschaft in Bonn regelmäßig anti-pakistanische Propaganda. ${ }^{171}$

$\mathrm{Zu}$ den größten südasienbezogenen Sorgen der bundesrepublikanischen Öffentlichkeit gehörte derweil das Schicksal der im neu entstandenen Staat Bangladesch verbliebenen Biharis. ${ }^{172}$ Bereits am 9. Februar 1972 hatte AA-Ministerialdirektor von Staden das westdeutsche Generalkonsulat in Dhaka nach einer entsprechenden Debatte im Auswärtigen Ausschuss des Bundestages angewiesen, „bei nächster Gelegenheit an möglichst hoher Stelle der Regierung von Bangladesh die Besorgnis der Bundesregierung über das Schicksal der nichtbengalischen Minderheiten in Bangladesh auszudrücken.“ Der Staatssekretär schlug außerdem vor, darauf hinzuweisen, „daß die deutsche Öffentlichkeit während der Auseinandersetzungen im vergangenen Jahr in gleicher Weise um das Schicksal der bengalischen Bevölkerung besorgt war. "173 In der indischen Botschaft in Bonn wiederum betrachtete man dieses Vorhaben mit Sorge und bemühte sich, die bundesrepublikanischen Bedenken gegenüber dem indischen Protegé Bangladesch zu zerstreuen. ${ }^{174}$

Die pakistanische Regierung andererseits kümmerte vor allem das Schicksal ihrer Soldaten, die nach Kriegsende noch in Indien festgehalten wurden.

169 Brief Diehls an die indische Unterhausabgeordnete Savitri Shyam, 3. Februar 1972, PA AA, B 37, Bd. 666.

170 Diehl an AA, 9. April 1972, PA AA, B 37, Bd. 666.

171 Müller an StS Frank, 11. April 1972, PA AA, B 37, Bd. 666.

172 Vgl. Hermann Schmitt-Vockenhausen an Walter Scheel, 29. Dezember 1971, PA AA, B 30, Bd. 671.

173 v. Staden an Generalkonsulat Dacca, 9. Februar 1972, PA AA, DHAK, Bd. 9089.

174 „Political Report for the Month of February 1972“ von D. Mukharji, 7. März 1972, NAI, Ministry of External Affairs, H I/1012(49)/72. 
Ende März 1972 forderte die pakistanische Botschaft in Bonn die Bundesregierung dazu auf, sich offiziell gegen die von der indischen Regierung angekündigte Auslieferung einiger pakistanischer Kriegsgefangener als Kriegsverbrecher an Bangladesch auszusprechen. ${ }^{175}$ In der Bonner AA-Zentrale besannen westdeutsche Diplomaten sich nun allerdings wieder ihrer neutralen Haltung im indischpakistanischen Dauerkonflikt und empfahlen, dieser Bitte nicht nachzukommen. ${ }^{176}$ An die westdeutschen Vertretungen in Südasien erging entsprechend Weisung, einschlägigen Bitten um Einflussnahme in diesem oder jenem Sinne eine Absage zu erteilen. Man hielt es „nicht für zweckdienlich, [...] in diese delikate Angelegenheit einzugreifen. “ ${ }^{177}$

Im Ost-Berliner MfAA suchte man derweil noch nach einer Erklärung für den indischen Sinneswandel und den durchwachsenen Erfolg des Winzer-Besuchs in Neu-Delhi Mitte Januar 1972. Von DDR-Diplomaten zur Rede gestellt, gab der indische Generalkonsul in Ost-Berlin J. C. Ajmani Ende Januar 1972 entschuldigend an, „[n]achdem Indien fast 23 Jahre mit der diplomatischen Anerkennung der DDR gewartet habe, möchte man offensichtlich nicht gerade in dem Augenblick, als sich in der BRD ein realistischerer Kurs durchsetzt, die BRD-Regierung und besonders W. Brandt verärgern.“ Er verwies nun auf den Mai 1972 als einen möglichen nächsten Termin für die Aufnahme diplomatischer Beziehungen. Nachdem Ajmani außerdem die angespannten indischen Beziehungen zu den USA erwähnte, warnte ihn sein Gesprächspartner Hans-Joachim Radde, Leiter der Abteilung Süd- und Südostasien im MfAA, vor der Annahme, „daß die BRDPolitik sich von der der USA grundsätzlich unterscheidet. “178

Dem westdeutschen AA war es inzwischen allerdings gelungen, die indische Regierung vom Gegenteil zu überzeugen, was nicht zuletzt der Person Willy Brandts zu verdanken war. Bereits während der bilateralen Regierungskonsultationen in Neu-Delhi im Januar 1972 glaubte Staatssekretär Paul Frank beobachtet zu haben, dass „das persönliche Ansehen des Bundeskanzlers dort ein politischer Faktor allerersten Ranges geworden ist. “179 Auch Botschafter Diehl fand es später „bemerkenswert, daß die DDR und ihre Lobby in Indien sich mit unmittelbaren

175 Botschaft Pakistans in der Bundesrepublik Deutschland an AA, 29. März 1972, PA AA, B 37, Bd. 692.

176 Vermerk von Berendonck, 13. April 1972, PA AA, B 37, Bd. 692.

177 Van Well an Botschaften Neu-Delhi, Dacca und Rawalpindi, 12. April 1972, PA AA, B 37, Bd. 692.

178 „Vermerk über ein Gespräch des Gen. Dr. Radde mit dem Leiter des indischen Generalkonsulats in der DDR, J. C. Ajmani, am 27.1.1972“ von Reder, 1. Februar 1972, PA AA, M 1, C 299/75. 179 „Kurzprotokoll 43. Sitzung des Ausschusses für wirtschaftliche Zusammenarbeit am Donnerstag, dem 27. Januar 1972, 9.30 Uhr in Bonn, Bundeshaus“, PA AA, B 37, Bd. 666. 
Angriffen gegen den Bundeskanzler im Allgemeinen zurückhalten, da sie hiermit wegen der Popularität Willy Brandts in Indien wenig Verständnis finden. “180 Ende Mai 1972 hieß es auch aus dem indischen Außenministerium, Indira Gandhi habe „große Hochachtung vor der Person und der Politik des Bundeskanzlers“ und „werde entsprechend ihrer in Bonn gegebenen Zusage den Bundeskanzler konsultieren, ehe endgültige Entscheidungen [über die DDR-Anerkennung] getroffen werden." ${ }^{181}$

Die DDR-Diplomaten waren von der Entwicklung der Dinge im Frühjahr 1972 sehr enttäuscht. Sie glaubten mittlerweile, mit einer Anerkennung der DDR durch Indien sei kaum mehr vor dem Herbst $1972 \mathrm{zu}$ rechnen. ${ }^{182}$ Dennoch versuchte man in den nächsten Monaten weiterhin, aus der solidarischen Haltung der DDR im Konflikt um Ostpakistan Kapital zu schlagen. Ende Mai 1972 brachte Herbert Fischer dem indischen Außenminister Swaran Singh gegenüber sein Unverständnis darüber zum Ausdruck, dass zwar die DDR auf Wunsch Indiens Bangladesch anerkannt habe, „man sich jedoch in Indien seines Freundes in der Not nicht mehr so gut zu erinnern“ scheine. Swaran Singh ließ sich hiervon allerdings kaum beeindrucken. ${ }^{183}$

Ungeahnte Hilfe erhielt Herbert Fischer unterdessen vom indischen Generalkonsul in der DDR J. C. Ajmani. Dieser schrieb Mitte Mai 1972 an das indische Außenministerium: „The time is now ripe for granting recognition to the GDR.“ Obwohl er später feststellen sollte, dass diese in erster Linie dem Ziel gedient hatte, Indien $\mathrm{zu}$ beeindrucken, ${ }^{184}$ verwies er dabei explizit auch auf die Solidarität, die die DDR Indien im Bangladeschkrieg erwiesen hatte und die von indischer Seite nicht ausreichend honoriert worden sei. ${ }^{185}$

Im folgenden Monat wurde DDR-Außenminister Otto Winzer im Gespräch mit dem indischen Minister für Wissenschaft und Technik, Chidambaram Subramaniam, weiter vertröstet. Wie seine Vorredner betonte Subramaniam zwar, die Anerkennung der DDR durch Indien sei ,im Prinzip beschlossen“, wichtiger als eine Normalisierung der Beziehungen zur DDR sei für die indische Regie-

180 Diehl an AA, 9. April 1972, PA AA, B 37, Bd. 666.

181 Behrends an AA, 26. Mai 1972, PA AA, B 37, Bd. 666.

182 „Niederschrift über ein Gespräch des Genossen Dr. Radde mit Gen. Fomin, Leiter der Abt. Südasien, am 19.1.1972 im MID“ von Elsner, 14. Februar 1972, PA AA, M 1, C 1044/77.

183 „Vermerk über ein Gespräch mit Außenminister Swaran Singh am 24. 5. 1972“ von Herbert Fischer, 25. Mai 1972, PA AA, M 1, C 299/75.

184 „Annual Political and Economic Report for 1972“ von J. C. Ajmani, 9. April 1973, NAI, Ministry of External Affairs, H I/1011(100)/73.

185 J. C. Ajmani an A. P. Venkateswaran/MEA, 19. Mai 1972, NAI, Ministry of External Affairs, H $\mathrm{I} / 1012(82) / 72$. 
rung allerdings die Entspannung der Lage in ganz Europa. Offenbar verknüpfte die indische Regierung außerordentliche Hoffnungen mit der Person des aufgrund eben jener Neuen Ostpolitik innenpolitisch angegriffenen Bundeskanzlers: „Wenn Brandt gestürzt werde, würde der Entspannungsprozess in Europa gehemmt, wenn nicht gar rückgängig gemacht“, war die Einschätzung des indischen Ministers und selbst der DDR-freundliche J. C. Ajmani stimmte darin mit ihm überein. ${ }^{186}$ Im Übrigen, so gestand der stellvertretende Außenminister Indiens öffentlich im Parlament ein, spielten auch ökonomische Erwägungen bei der Entscheidung über die DDR-Anerkennung eine zentrale Rolle. ${ }^{187}$ Noch immer hielt die indische Regierung also die Furcht vor etwaigen entwicklungspolitischen Vergeltungsmaßnahmen der BRD von der grundsätzlich befürworteten DDR-Anerkennung ab.

Bundeskanzler Willy Brandt bewies im Umgang mit der indischen Regierungschefin zudem tatsächlich außerordentliches Fingerspitzengefühl. Nachdem Indira Gandhi ihm Ende Mai 1972 ihre Bewunderung für den Erfolg seiner Ostpolitik aussprach, dabei aber auch darauf hinwies, dass sie die DDR-Anerkennung nicht mehr allzu lange würde aufschieben können, ${ }^{188}$ reagierte Willy Brandt ausgesucht höflich. Er dankte Indira Gandhi für ihre verständnisvolle und konstruktive Haltung zur Anerkennung der DDR, die die Entspannung in Mitteleuropa „und ihre Ausstrahlung auf die Weltpolitik“ entscheidend unterstützt habe. ${ }^{189}$ Dieses Lob verfehlte seine Wirkung in Indien nicht. Im Zuge der Verhandlungen zum Vertrag von Shimla deutete der indische Diplomat D. P. Dhar später sogar an, der deutsch-polnische Vertrag könne „ein Modell auch für die Lösung der Kaschmirfrage sein. “190

Als die DDR-Diplomaten einsahen, dass die indische Regierung die Entspannungspolitik Bundeskanzler Willy Brandts keinesfalls gefährden wollte, vollzogen sie eine radikale argumentative Wende. Mitte August 1972 behauptete DDR-Generalkonsul Herbert Fischer im Gespräch mit dem indischen Außenminister Swaran Singh, eine Anerkennung der DDR durch Indien wäre der deutschdeutschen Entspannung unbedingt förderlich und könnte dem westdeutschen Bundeskanzler Willy Brandt innenpolitisch den Rücken stärken. ${ }^{191}$ Swaran Singh

186 Vermerk von Kirchhof, 28. Juni 1972, PA AA, M 1, C 299/75.

187 Monatsbrief von Kawretzke an Oskar Fischer, 25. Mai 1972, PA AA, M 1, C 1730/76.

188 AA / IfZ: $A A P d B, 1972$, Bd. 2, Dok. 177, Anm. 7.

189 Ebd., Anm. 8; s. auch Willy Brandt an Indira Gandhi, 22. Juni 1972, NMML, P. N. Haksar Papers (III Installment), Subject File No. 182.

190 Behrends an AA, 4. Juli 1972, BArch, B 122/12493.

191 Vermerk von Herbert Fischer, 22. August 1972, PA AA, M 1, C 299/75. So Fischer auch bereits drei Monate zuvor im Gespräch mit Swaran Singh, s. „Vermerk über ein Gespräch mit Außenmi- 
erkannte jedoch, dass dies den Wünschen der Bundesregierung diametral widersprach und wollte die Anerkennung der DDR daher noch nicht fest zusagen. ${ }^{192} \mathrm{Im}$ Rahmen der deutsch-deutschen Verhandlungen beklagten Otto Winzer und Erich Honecker daraufhin wiederholt die Aktivitäten der Bundesregierung gegen eine DDR-Anerkennung durch Indien. ${ }^{193}$

\section{Indien erkennt die DDR an}

Anfang September 1972 war es soweit. Indira Gandhis Vertrauter P. N. Haksar versprach Herbert Fischer ganz konkret, mit der lang ersehnten DDR-Anerkennung sei nunmehr Ende des Monats bzw. Anfang Oktober $1972 \mathrm{zu}$ rechnen. Anders als von Fischer vermutet, erfolgte der indische Sinneswandel allerdings nicht als Vergeltungsmaßnahme für die kürzlich bekannt gewordene und aus indischer Sicht bedrohlich wirkende Annäherung zwischen der BRD und der VR China. ${ }^{194}$ Die indische Entscheidung war vielmehr ein Ergebnis der erfolgreichen Verhandlungen zum deutsch-deutschen Grundlagenvertrag.

Am Abend des 19. September 1972 teilte der bundesrepublikanische Chefunterhändler der deutsch-deutschen Verhandlungen Egon Bahr dem indischen Botschafter Kewal Singh bei einem geheimen Treffen (von dem selbst das Auswärtige Amt nicht erfahren sollte) mit, die indische Regierung sei nunmehr frei, über den Zeitpunkt der DDR-Anerkennung nach eigenem Ermessen zu entscheiden. Die Bundesregierung werde diesen Schritt nicht mehr als gegen sie gerichtet missverstehen. Für Kewal Singh kam diese Aussage des wichtigsten Beraters Willy Brandts einer Stellungnahme des westdeutschen Bundeskanzlers selbst gleich. ${ }^{195}$ Nach diesem Gespräch zwischen ihrem Botschafter und Egon Bahr informierte Indira Gandhi den Bundeskanzler am 30. September darüber, dass Indien die DDR am 7. Oktober 1972 anerkennen würde. ${ }^{196}$

nister Swaran Singh am 24. 5. 1972“ von Herbert Fischer, 25. Mai 1972, PA AA, M 1, C 299/75; vgl. auch Diehl an AA, 9. April 1972, PA AA, B 37, Bd. 666.

192 Vermerk von Herbert Fischer, 22. August 1972, PA AA, M 1, C 299/75.

193 AA / IfZ: $A A P d B$, 1972, Bd. 2, Dok. 191; Ebd., Dok. 263.

194 Monatsbrief von Herbert Fischer an Wolfgang Kiesewetter, 12. September 1972, PA AA, M 1, C 1730/76; so allerdings auch Voigt: Die Indienpolitik der DDR, S. 667.

195 Kewal Singh an P. N. Haksar, 20. September 1972, NMML, P. N. Haksar Papers (I-II Installment), Subject File No. 54.

196 Indira Gandhi an Willy Brandt, 30. September 1972, NMML, P. N. Haksar Papers (I-II Installment), Subject File No. 54; eingegangen bei Brandt am 2. Oktober 1972, s. AA / IfZ: $A A P d B, 1972$, Bd. 3, Dok. 304, Anm. 7. 
Am 3. Oktober benachrichtigte das indische Außenministerium auch den DDR-Generalkonsul Herbert Fischer über diese Entscheidung. ${ }^{197}$ Zur Erklärung hieß es dazu von indischer Seite in den folgenden Tagen, die deutsch-deutschen Verhandlungen zum Grundlagenvertrag seien nunmehr faktisch abgeschlossen, die Zeit also reif für eine diplomatische Anerkennung der DDR. ${ }^{198}$ Eine sowjetische Einschätzung von Mitte September 1972, mit der DDR-Anerkennung durch Indien sei vor den westdeutschen Bundestagswahlen am 19. November 1972 nicht $\mathrm{zu}$ rechnen, ${ }^{199}$ war damit widerlegt.

Obwohl Egon Bahr stellvertretend für Bundeskanzler Willy Brandt sein Einverständnis dazu erklärt hatte, empfand man die indische Ankündigung im Auswärtigen Amt wiederum als Affront. Am 4. Oktober 1972 traf der westdeutsche Außenminister Walter Scheel in New York seinen indischen Amtskollegen Swaran Singh und machte deutlich, dass die innerdeutschen Verhandlungen ganz im Gegenteil keineswegs bereits abgeschlossen seien und eine Anerkennung der DDR durch Drittstaaten deshalb weiterhin unerwünscht sei. Scheel bat um einen weiteren Aufschub des angekündigten Termins, vor allem aber darum, die DDR nicht gerade an ihrem Nationalfeiertag anzuerkennen. ${ }^{200}$ Zwei Tage später unterstrich auch Willy Brandt in einem persönlichen Brief an Indira Gandhi noch einmal diesen Wunsch seines Außenministers. ${ }^{201}$

Tatsächlich ließ die indische Regierung sich noch ein letztes Mal von der Bundesregierung beeindrucken. ${ }^{202}$ Kaum hatte die indische Premierministerin das Schreiben des Bundeskanzlers gelesen, wurde die eigentlich bereits beschlossene DDR-Anerkennung kurzfristig doch noch einmal verschoben. Als Zeitpunkt legte Indira Gandhi nun den Morgen des 8. Oktober 1972 um 6.00 Uhr Ortszeit fest. ${ }^{203}$ Außerdem kam sie der Bitte Brandts nach, die öffentliche Mitteilung über die DDR-Anerkennung um den Zusatz zu erweitern, dass die indische Entschei-

197 „Telegramm von Fischer (New Delhi)“, 3. Oktober 1972, PA AA, M 1, C 1488/73.

198 J. C. Ajmani an A. P. Venkateswaran/MEA, 4. Oktober 1972, NAI, Ministry of External Affairs, H I/1012(82)/72; vgl. auch P. N. Haksar an Kewal Singh, 2. Oktober 1972, NMML, P. N. Haksar Papers (I-II Installment), Subject File No. 54 und Radde an Herbert Fischer, 6. Oktober 1972, PA AA, M 35, ZR 1009/03.

199 „Vermerk über ein Gespräch des Gen. Thun mit Gen. Smirnow (Abt. Südasien) im MID am 20.9.1972“, 26. September 1972, PA AA, M 1, C 1763/76.

200 AA / IfZ: $A A P d B, 1972$, Bd. 3, Dok. 311.

201 Willy Brandt an Indira Gandhi, 6. Oktober 1972, NMML, P. N. Haksar Papers (I-II Installment), Subject File No. 54.

202 P. N. Haksar an Kewal Singh, 6. Oktober 1972, NMML, P. N. Haksar Papers (I-II Installment), Subject File No. 54.

203 Herbert Fischer an Florin, 6. Oktober 1972, PA AA, M 35, ZR 1313/85. 
dung, die Souveränität der DDR anzuerkennen, keinesfalls eine künftige, friedliche, deutsche Wiedervereinigung ausschließen solle. ${ }^{204}$ Indien war damit nach Jugoslawien (1957) und Ägypten (1969) der dritte bedeutende bündnisfreie Staat und insgesamt das 32. Land, mit dem die DDR diplomatische Beziehungen aufnahm. ${ }^{205}$

Nachdem die indische Regierung die Anerkennung der DDR öffentlich ausgesprochen hatte, zeigte man sich in der DDR-Vertretung in Neu-Delhi erleichtert, wie Herbert Fischer dem stellvertretenden DDR-Außenminister Oskar Fischer später berichtete: „Nach dem ständigen Hin und Her, Versprechungen, Hoffnungen, und dann wieder Verzögerungen, Enttäuschungen löste die tatsächliche Herstellung diplomatischer Beziehungen bei uns allen Freude und bei vielen auch Tränen der Freude aus.“206

Die Tatsache allerdings, dass die bundesrepublikanische Seite sich mit ihrer Forderung hatte durchsetzen können, der offiziellen Anerkennungserklärung eine Formulierung zur deutschen Wiedervereinigung beizufügen, bedeutete für Herbert Fischer dann doch einen gewissen Wermutstropfen. Nachdem außerdem der indische Staatssekretär im Außenministerium T. N. Kaul unumwunden zugab, dass auch die letzte Verschiebung des lang ersehnten Anerkennungstermins aus Rücksichtnahme gegenüber der Bundesregierung erfolgt war, ${ }^{207}$ mahnte Fischer, „daß wir in unseren Anstrengungen keinesfalls nachlassen dürfen, weil der Feind noch stärker gegen uns arbeiten wird und weil wir uns auch nicht auf die Standhaftigkeit unserer indischen Freunde verlassen können.“208

\section{Die DDR und Pakistan}

Am 17. Oktober 1972, nur gut eine Woche nach der indischen DDR-Anerkennung, sprach auch der pakistanische Botschafter im Bonner AA vor, um sich über die „deutschlandpolitischen Auswirkungen der Anerkennung der DDR durch

204 P. N. Haksar an Kewal Singh, 6. Oktober 1972, NMML, P. N. Haksar Papers (I-II Installment), Subject File No. 54; AA / IfZ: AAPdB, 1972, Bd. 3, Dok. 311, Anm. 5. Die Bundesregierung hatte schon im Sommer 1969 empfohlen, bei einer Anerkennung der DDR durch Drittstaaten auf eine Wiedervereinigungsklausel zu bestehen, s. Vermerk Egon Bahr, 1. Juli 1969, PA AA, B 150, Bd. 156. 205 Jacobsen, Hans-Adolf, Gert Leptin, Ulrich Scheuner und Eberhard Schulz (Hrsg.): Drei Jahrzehnte Außenpolitik der DDR. Bestimmungsfaktoren, Instrumente, Aktionsfelder. München / Wien: R. Oldenbourg 1979, S. 857.

206 Monatsbrief von Herbert Fischer an Oskar Fischer, 17. Oktober 1972, PA AA, M 1, C 1730/76.

207 Herbert Fischer an Florin, 6. Oktober 1972, PA AA, M 35, ZR 1313/85.

208 Monatsbrief von Herbert Fischer an Oskar Fischer, 17. Oktober 1972, PA AA, M 1, C 1730/76. 
Indien“ zu informieren. Obwohl er beteuerte, dass er nicht mit einer Änderung der Haltung seiner eigenen Regierung zur Frage der DDR-Anerkennung rechne, ${ }^{209}$ mochten westdeutsche Diplomaten dies kaum glauben. Nach Einschätzung des bundesrepublikanischen Botschafters in Pakistan Norbert Berger hielt allein die Sorge um eventuelle Auswirkungen auf westdeutsche Entwicklungshilfezahlungen die pakistanische Regierung noch davon ab, die DDR ebenfalls anzuerkennen, ,wenn auch die eilige Anerkennung von Bangla Desh seinerzeit nicht gerade zu einer Erwärmung der Gefühle für die DDR beigetragen hat.“210

Nach den früheren bilateralen Kontakten zwischen Pakistan und der DDR war der Austausch zwischen den beiden Staaten während des Konflikts in und um Ostbengalen tatsächlich deutlich zurückgegangen. Pakistanische Annäherungsversuche im November $1971^{211}$ hatten sowjetische Diplomaten angesichts der klaren proindischen Positionierung der DDR abgetan: Die pakistanische Regierung „spiele“ nur mit der DDR, um einen Keil zwischen sie und Indien zu treiben. $^{212}$

Im Februar 1972 waren auch die westdeutschen Sorgen, die pakistanische Regierung könnte die unerwünschte Anerkennung Bangladeschs durch die Bundesregierung ihrerseits mit einer Anerkennung der DDR beantworten, unbegründet geblieben. Also bestanden im Frühjahr 1972 noch keinerlei offizielle diplomatische, konsularische oder auch nur Handelsbeziehungen zwischen Pakistan und der DDR. ${ }^{213}$ Selbst als die sowjetische Führung beim Besuch des pakistanischen Präsidenten Bhutto in Moskau Mitte März 1972 Druck hinsichtlich der Aufnahme diplomatischer Beziehungen mit der DDR auszuüben versuchte, zeigte dieser sich hierzu weiterhin nicht bereit. $^{214}$

Indem das pakistanische Außenministerium in Islamabad im Sommer 1972 verkündete, bis auf Weiteres keine Anträge auf die Gründung bilateraler Freundschaftsgesellschaften $\mathrm{zu}$ bearbeiten, verwehrte man der DDR-Führung außerdem ein bewährtes Mittel, um auf subtile Art mit anderen Staaten in Kontakt zu treten. ${ }^{215}$ Im August 1972 gründete sich in Lahore zwar dennoch eine „Pakistan-

209 Vermerk von Dr. Jesser, 17. Oktober 1972, PA AA, ISLA, Bd. 8149.

210 Berger an AA, 18. Oktober 1972, PA AA, B 37, Bd. 693.

211 S. etwa „Vermerk über ein Gespräch des Gen. Bittner mit dem Botschafter Pakistans, Marker, am 10.11.1971 in unserer Botschaft“ von Elsner, 15. November 1971, PA AA, M 1, C 1444/76.

212 „Vermerk über ein Gespräch des Gen. Lützkendorf mit Gen. Fomin, Leiter der Abt. Südasien, am 12.11.1971 im MID“ von Elsner, 16. November 1971, PA AA, M 1, C 1253/75.

213 Enzweiler an AA, 10. März 1972, PA AA, ISLA, Bd. 18539.

214 Vermerk von Gahlich, 4. April 1972, PA AA, M 1, C 91/78.

215 Ministry of Foreign Affairs Islamabad an die diplomatischen Vertretungen in Pakistan, Islamabad, 14. Juli 1972, PA AA, M 2, B 151/78. 
GDR Youth Friendship Society“, deren erklärtes Ziel die Aufnahme diplomatischer Beziehungen und der Austausch von Botschaftern zwischen der DDR und Pakistan war. ${ }^{216}$ Im Ost-Berliner MfAA bewertete man die Gesellschaft später jedoch als „eine typische letter head Vereinigung“ ohne ernstzunehmende Aktivitäten..$^{217}$

Eine wirkliche Änderung der pakistanischen Haltung zur DDR brachte wiederum erst die Anerkennung der DDR durch Indien. Am 21. Oktober 1972 teilte ein Vertreter des pakistanischen Außenministeriums dem westdeutschen Botschafter Berger in Islamabad mit, dass seine Regierung ,in naher Zukunft“ diplomatische Beziehungen mit der DDR aufzunehmen beabsichtige. Begründet wurde diese Entscheidung mit dem zunehmenden Druck der UdSSR und anderer sozialistischer Staaten sowie der öffentlichen Meinung in Pakistan. Im Übrigen habe die Bundesrepublik ,ja auch unter den ersten Bangla Desh anerkannt“, wie es hieß. ${ }^{218}$ Bergers Gegenrede, man habe doch erst am Vortag neue Kapitalhilfe- und Umschuldungsabkommen unterzeichnet, ${ }^{219}$ ließ sein Gegenüber nicht gelten. Seine Regierung könne mit der DDR-Anerkennung nicht mehr länger warten, „um den Anschluß nicht zu verlieren.“220

In der Bonner AA-Zentrale reagierte man auf diese Nachricht aus Islamabad prompt und entrüstet. Botschafter Berger wurde angewiesen, der pakistanischen Regierung umgehend und „auf möglichst hoher Ebene“ mitzuteilen, dass diese Ankündigung nur einen Tag nach Abschluss zweier wichtiger Abkommen in Bonn „erhebliche Irritation hervorgerufen“ habe. Vor der Anerkennung Bangladeschs habe die Bundesregierung die pakistanische Regierung zumindest konsultiert und ihre Entscheidung begründet. Von pakistanischer Seite erwarte man ein entsprechendes Verhalten. Andernfalls sei nicht auszuschließen, „daß auf die künftige Ausgestaltung der deutsch-pakistanischen Beziehungen, die bisher eng und freundschaftlich waren, ein Schatten fällt." ${ }^{221}$

Noch vor Unterzeichnung der zu den bereits vereinbarten Kapitalhilfe- und Umschuldungsabkommen gehörenden Protokolle sollte dies der pakistanischen

216 Mussadeq Ahad Shah (Präsident der Pakistan-GDR Your Friendship Society) an Walter Ulbricht, 25. August 1972, PA AA, M 2, B 151/78.

217 Bräsel an Köcher, 1. Dezember 1972, PA AA, M 2, B 151/78.

218 Berger an AA, 23. Oktober 1972, PA AA, ISLA, Bd. 8149. Vor diesem Zusammenhang hatte Berger schon Monate zuvor gewarnt, s. Berger an AA, 2. Februar 1972, PA AA, DHAK, Bd. 9092.

219 Dies war im Übrigen wenige Monate zuvor erstes Anliegen des pakistanischen Botschafters bei seinem Antrittsbesuch im Bonner AA gewesen, s. Vermerk von StS v. Braun, 17. Juli 1972, PA AA, B 2, Bd. 193.

220 Berger an AA, 23. Oktober 1972, PA AA, ISLA, Bd. 8149.

221 Diesel an Botschaft Rawalpindi, 23. Oktober 1972, PA AA, ISLA, Bd. 8149. 
Regierung mitgeteilt und darauf hingewiesen werden, man unterzeichne in der Erwartung, dass die pakistanische Regierung „,von dem angekündigten Schritt gegenüber der DDR Abstand nimmt, bis die Grundvertragsverhandlungen in der Sache abgeschlossen sind.“ Im Übrigen habe die Bundesregierung sich bei der indischen Botschaft auch für eine Rückführung pakistanischer Kriegsgefangener und Zivilisten eingesetzt, die sich seit Ende des Bangladeschkriegs in Gefangenschaft befanden.222 Am 25. Oktober 1972 wurde dies dem pakistanischen Botschafter in der Bonner AA-Zentrale auch noch einmal persönlich dargelegt. Dieser versprach zwar, seiner Regierung entsprechend zu berichten, wies allerdings auch darauf hin, dass Pakistan nunmehr als einziges Land des indischen Subkontinents noch keine diplomatischen Beziehungen zur DDR unterhielt. ${ }^{223}$

Die Bonner Bemühungen zeigten Wirkung: Derselbe pakistanische Regierungsvertreter, der Botschafter Berger bereits am 21. Oktober 1972 aufgesucht hatte, teilte ihm wenige Tage später beschwichtigend mit, der „Ausdruck ,in naher Zukunft' bedeute nicht, dass die Anerkennung ,übermorgen’ erfolgen würde.“224 Stattdessen war nun die Rede davon, mit einer Anerkennung der DDR durch Pakistan sei in einigen Wochen zu rechnen, ${ }^{225}$ vermutlich erst nach der Bundestagswahl am 19. November 1972. ${ }^{226}$ Eine die pakistanische DDR-Anerkennung bedauernde Pressemitteilung hatte man im AA zu diesem Zeitpunkt bereits entworfen. ${ }^{227}$

Am 11. November 1972 schließlich ließ der pakistanische Botschafter in Bonn den AA-Staatssekretär Paul Frank wissen, dass die DDR-Anerkennung am 15. November 1972 erfolgen würde. ${ }^{228}$ Er betonte, dass seine Regierung damit wie zuvor angekündigt ${ }^{229}$ den Abschluss der Verhandlungen über den deutschdeutschen Grundvertrag abgewartet hätte. Staatssekretär Frank bedauerte daraufhin zwar, dass die pakistanische Regierung nicht auch noch die bevorstehende Bundestagswahl abwarten würde, bedankte sich im Übrigen aber für das

222 Van Well an Botschaft Rawalpindi, 24. Oktober 1972, PA AA, ISLA, Bd. 8149.

223 Diesel an Botschaft Rawalpindi, 25. Oktober 1972, PA AA, ISLA, Bd. 8149.

224 Berger an AA, 26. Oktober 1972, PA AA, ISLA, Bd. 8149.

225 ,Vermerk über ein Gespräch des Gen. Thun mit Gen. Fomin (Leiter der Abteilung Südasien des MID) am 25. Oktober 1972“ von Scholz, 3. November 1972, PA AA, M 1, C 97/78; von der bundesrepublikanischen Intervention erfuhr die DDR Anfang November 1972 durch ungarische Diplomaten, s. Vermerk von Peters, 15. November 1972, PA AA, M 1, C 97/78.

226 Ritter an Walter Scheel, 30. Oktober 1972, PA AA, B 37, Bd. 693.

227 Thönnes an Ref. 103, 25. Oktober 1972, PA AA, B 37, Bd. 693.

228 Hauthal an Botschaft Rawalpindi, 11. November 1972, PA AA, B 37, Bd. 693.

229 Vgl. Berger an AA, 1. März 1973, PA AA, B 37, Bd. 100297. 
pakistanische Entgegenkommen. ${ }^{230}$ Im MfAA der DDR erfuhr man unmittelbar danach ebenfalls vom beabsichtigten pakistanischen Schritt. ${ }^{231}$

Diplomaten der Botschaften der DDR und Pakistans in Moskau verhandelten daraufhin die genaue Formulierung eines gemeinsamen Kommuniqués anlässlich der Herstellung diplomatischer Beziehungen. ${ }^{232}$ Das sowjetische Außenministerium drängte derweil zur Eile. Der Austausch von Botschaftern zwischen Pakistan und der DDR war der Moskauer Führung ein sehr willkommenes Mittel, den befürchteten zunehmenden chinesischen Einfluss in Pakistan zurückzudrängen. ${ }^{233}$ Am 15. November 1972 war es dann schließlich soweit und auch Pakistan erkannte die DDR offiziell völkerrechtlich an. ${ }^{234}$

\subsection{Zwischenbilanz}

Für die beteiligten Staaten des indischen Subkontinents bedeutete der Bangladeschkrieg eine fundamentale außenpolitische Neuorientierung. Nach dem siegreichen Ende des kurzen Dritten Indisch-Pakistanischen Krieges konnte Indien sich seiner Stellung als regionale Vormacht in Südasien endlich sicher sein, während der Kriegsausgang für Pakistan den Beginn einer stärkeren Wendung hin zur arabischen Welt markierte. Bangladesch wiederum stand nach Erlangen der staatlichen Unabhängigkeit vor der Herausforderung, sich nicht allein von den pakistanischen Unterdrückern, sondern auch von seinen indischen Befreiern abzugrenzen. Außerdem galt es für den jüngsten südasiatischen Staat, neben einer nationalen Identität auch noch ein funktionierendes Staatswesen zu entwickeln.

Auch die Großmächte mussten sich nach dem Bangladeschkrieg ihrer Positionen in Südasien neu vergewissern. Der US-Administration gelang es nur sehr langsam, das während des Krieges verspielte Vertrauen in Indien zurückzugewinnen, während die Moskauer Führung die Sorge umtrieb, ihre neuen chinesischen Widersacher könnten ihnen im Streit um Einfluss im strategisch weiterhin wichtigen Pakistan den Rang ablaufen. In Indien wiederum erfuhr die UdSSR zwar

230 Hauthal an StS Frank, 13. November 1972, PA AA, B 37, Bd. 693.

231 „Aufnahme diplomatischer Beziehungen DDR - Pakistan“ von Krüger, 12. November 1972, PA AA, M 92, ZR 4106/14.

232 ,Vermerk über ein Gespräch des Gen. Thun mit dem Geschäftsträger der Botschaft Pakistans in der UdSSR, M. A. Khairi, am 13.11.1972“"von Scholz, 15. November 1972, PA AA, M 1, C 97/78. 233 „Mitteilung des Genossen Thun, Moskau, am 15.11.1972, 7.45 Uhr, über WTSCH“, aufgenommen von H. Haase, 15. November 1972, PA AA, M 1, C 97/78.

234 Berger an AA, 1. März 1973, PA AA, B 37, Bd. 100297. 
große Dankbarkeit für die während des Krieges gewährte Solidarität, doch führte diese punktuelle Kooperation nicht automatisch zu einer längerfristigen sicherheitspolitischen Allianz zwischen den beiden Ländern. Denn auch der Dritte Indisch-Pakistanischen Krieg, der allzu leicht zu einem Krieg der Großmächte hätte werden können, sollte nichts am grundsätzlichen Postulat der indischen Bündnisfreiheit ändern. ${ }^{235}$ Vielmehr suchte die indische Regierung nach der indisch-sowjetischen Annäherung des Jahres 1971 im Folgejahr vermehrt den ausgleichenden Kontakt zu Staaten des westlichen Bündnisses. Hieraus erklärte sich Anfang 1972 auch der Wunsch der indischen Regierung, nicht allein sozialistische Staaten mögen ihren Schützling Bangladesch anerkennen. ${ }^{236}$

Der Bangladeschkrieg sollte sich im Laufe des Jahres 1972 als ein Katalysator der deutsch-deutschen Südasienpolitik erweisen. Waren es bis zum Kriegsausbruch Anfang Dezember 1971 noch die südasiatischen Konfliktparteien, die die beiden deutschen Staaten umgarnten und um Unterstützung baten, so drehte sich dieses Verhältnis nach Ende des Krieges sehr schnell um. Vor allem die DDRFührung in Ost-Berlin hoffte, ihre in diesem Konflikt gezeigte Solidarität mit der Regierung Indira Gandhi könnte endlich den lang erhofften Durchbruch bringen und würde von der indischen Premierministerin durch eine völkerrechtliche Anerkennung honoriert.

Zum „Eisbrecher“ in Südasien bestimmten DDR-Außenpolitiker die Aufnahme diplomatischer Beziehungen mit Bangladesch. Noch während der Kampfhandlungen in und um Ostbengalen hatte die provisorische Regierung von Bangladesch weltweit nach Verbündeten gesucht. Mit ihrem Versprechen gegenseitiger Anerkennung nach Erlangung staatlicher Unabhängigkeit war sie in der DDR fündig geworden. Im MfAA nahm man die unerfahrene politische Führung des jungen Staates beim Wort, und es gelang dem damaligen DDR-Außenminister Otto Winzer im ehemaligen Ostpakistan erfolgreich, während eines persönlichen Besuchs Mitte Januar 1972 bei seinem dortigen Amtskollegen Abdus Samad Azad und dem ersten Premierminister des unabhängigen Bangladesch Mujibur Rahman die Zusage einer gegenseitigen Anerkennung zu erwirken. Auch bundesrepublikanische Interventionsversuche konnten diese Entwicklung nicht mehr verhindern. In bis dahin ungeahnter Weise waren die DDR-Diplomaten außerdem dazu in der Lage gewesen, sich gegenüber der Moskauer Führung durchzusetzen und ihre Zustimmung zur Anerkennung von Bangladesch zu erhalten.

235 Vgl. Diehl an AA, 25. Januar 1972, PA AA, B 30, Bd. 654.

236 S. hierzu auch Das Gupta: Indiens sicherheitspolitische Wende zwischen 1962 und 1974, S. $114-5$. 
Während Otto Winzer auf die Signalwirkung dieses Schrittes für die indische Regierung hoffte, fürchtete man genau dies im Bonner AA. Es galt also, der indischen Regierung ein verlockendes Gegenangebot zu machen, um sie von der unliebsamen DDR-Anerkennung abzuhalten. Ein solches fand die Bundesregierung schließlich mithilfe ihrer europäischen Partner. Noch mehr als zwei Monate vor der Anerkennung Bangladeschs durch die Nixon-Administration, war deren Verhalten für die Bundesregierung kaum richtungsweisend. Im Gegenteil: Sie profitierte sogar von der indisch-amerikanischen Entfremdung während des Bangladeschkriegs und konnte einspringen, als die USA für Indien kurzfristig als politische Partner und Entwicklungshilfegeber ausfielen. Ihre gemeinsame Blockzugehörigkeit zum westlichen Staatenbündnis diente der Bundesregierung vielmehr allein dann als Argument, wenn es ihren eigenen Interessen entsprach. Etwa als pakistanische Diplomaten andeuteten, die DDR anerkennen zu wollen.

Das Inaussichtstellen einer Anerkennung Bangladeschs gemeinsam mit den anderen EWG-Staaten erwies sich als genialer Schachzug der Bundesregierung: Als Lockmittel gegenüber Indien war das Angebot einer gleich mehrfachen Anerkennung Bangladeschs durch westliche Staaten zu attraktiv, als dass die indische Regierung es durch ein Nachgeben gegenüber dem DDR-Drängen hätte gefährden wollen. Gleichzeitig war das Handeln im Verbund auch ein ausreichend abschreckendes Druckmittel gegenüber der pakistanischen Führung, die vielleicht die Bundesregierung allein, sicherlich jedoch nicht auch ihre EWG-Partner (zumal Mitglieder des Pakistan-Konsortiums) für die frühe Anerkennung der Unabhängigkeit seines ehemaligen Ostflügels abgestraft hätte. Dadurch und dank des hohen Ansehens, das Bundeskanzler Willy Brandt in Indien genoss, konnte die Bundesregierung die diplomatische Anerkennung der DDR dort noch bis zu einem ihr genehmeren Zeitpunkt im Herbst 1972 hinauszögern.

Im Umgang mit Pakistan hatte die DDR-Führung im November 1972 größeres Glück. Die pakistanische Regierung um Zulfikar Ali Bhutto trug ihr weder die proindische Haltung während des Krieges im Vorjahr noch die rasche Anerkennung des früheren pakistanischen Ostflügels nach. Wie für die indische Regierung, so bedeutete die Anerkennung der DDR auch für die Regierung Bhutto lediglich einen zeitgemäßen Schritt, der den gewandelten Vorzeichen des deutsch-deutschen Verhältnisses Rechnung trug. Dass das sowjetische Außenministerium hierbei gleichsam die Schirmherrschaft übernahm, erleichterte den pakistanischen Außenpolitikern ihre Entscheidung, die DDR nach einer letzten kurzen Verzögerung durch die Bundesregierung Mitte November 1972 anzuerkennen. 
